\title{
Robust pricing for airlines with partial information
}

\author{
Bo Feng ${ }^{1}$ (D) Jixin Zhao ${ }^{1} \cdot$ Zheyu Jiang $^{2}$
}

Accepted: 26 December 2020 / Published online: 26 February 2021

(c) The Author(s), under exclusive licence to Springer Science+Business Media, LLC part of Springer Nature 2021

\begin{abstract}
In the spot market for air cargo, airlines typically adopt dynamic pricing to tackle demand uncertainty, for which it is difficult to accurately estimate the distribution. This study addresses the problem where a dominant airline dynamically sets prices to sell its capacities within a two-phase sales period with only partial information. That partial information may show as the moments (upper and lower bounds and mean) and the median of the demand distribution. We model the problem of dynamic pricing as a distributional robust stochastic programming, which minimizes the expected regret value under the worst-case distribution in the presence of partial information. We further reformulate the proposed non-convex model to show that the closed-form formulae of the second-stage maximal expected regret are well-structured. We also design an efficient algorithm to characterize robust pricing strategies in a polynomial-sized running time. Using numerical analysis, we present several useful managerial insights for airline managers to strategically collect demand information and make prices for their capacities in different market situations. Moreover, we verify that additional information will not compromise the viability of the pricing strategies being implemented. Therefore, the method we present in this paper is easier for airlines to use.
\end{abstract}

Keywords Robust pricing - Distributional robust stochastic programming - Air cargo · Partial information

\section{Introduction}

The air cargo industry has played an indispensable role in goods transportation and international trade in recent decades. Boeing (2018) projected that the annual industry-wide growth rate in terms of freight tonne kilometers (FTKs) would be $4.2 \%$ over the next two decades, which would increase revenue from 256 billion revenue ton kilometers (RTKs) in 2017 to 584 billion RTKs in 2037 (Boeing 2018). Such a remarkable growth rate is not surprising because air cargo offers incomparable advantages in urgent mid- to long-distance

Bo Feng

neu_fengbo@163.com

1 School of Business and Research Center for Smarter Supply Chain, Soochow University, Suzhou 215021, China

2 Miami Business School, University of Miami, Coral Gables, FL 33146, USA 
shipping (Lange 2019). The reliability and efficiency of air freight are difficult to replicate using other means of transportation, given the increasing demand for high-speed, highsecurity, and low-risk transport.

Despite its rapid expansion, the air cargo industry is not immune to the problems associated with demand uncertainty. The full-year 2019 data released by the International Air Transport Association (IATA) show that industry-wide FTKs fell by 3.3\% in 2019. This can be attributed to several factors, including stagnant global trade, sluggish GDP growth in manufacturing-intensive economies, and declining export orders. The current year is proving to be another challenging year for the air cargo business, with demand slumping as the spread of COVID-19. Based on data released by the IATA in February 2020, adjusted demand, measured in cargo ton kilometers, fell by $9.1 \%$ compared with the same period in 2019. However, demand for pharmaceuticals is rising sharply, with shipments now double those of last year.

In air cargo revenue management, most pricing decisions dealing with uncertainty remain unsatisfactory, partly because of limited information. This incomplete information acquisition is attributed to distinct characteristics of the air cargo industry. Air cargo transport involves multiple participants, complex processes, intricate transportation networks, and multiple freight dimensions (Bartodziej et al. 2009; Leung et al. 2009; Wang and Kao 2008). The considerable volatility that exists in the booking process creates high levels of uncertainty in capacity availability. Cargo capacity is further determined by various factors, including pivot weight, volume, and type (Leung et al. 2009). Moreover, air cargo can be transshipped via multiple itineraries between an origin and destination (Amaruchkul et al. 2011). These characteristics introduce more complexity and uncertainty to the task of accessing and processing air freight data. Therefore, airlines are required to strategically adjust their prices to overcome the problem of limited information.

In this study, we consider a setting where a dominant airline in a regional market dynamically adjusts its prices to sell its total capacities within a two-phase sales period. In operational practices, most airlines operate within a so-called hub-and-spoke network, wherein a dominant player commonly exists in each regional market (Feng et al. 2015). A good example of the hub-and-spoke structure is provided by Delta Airlines, whose hub is located at Hartsfield-Jackson Atlanta International Airport. Delta has achieved oligopolistic (if not monopolistic) control and regional market dominance because it assumes the dominance of the hub. As a consequence, pricing operates under a leader-follower model; that is, the dominant airline makes its pricing decisions, and the other airlines follow its prices (Feng et al. 2015).

We model the dynamic pricing problem as a distributional robust stochastic programming (DRSP) with an uncertainty set that gives moments and quantiles of the random factor (the random part of the stochastic demand function). Instead of maximizing overall profitability, the model minimizes the expected regret value under the worst-case distribution. The regret value is the revenue gap from not making the best decision; that is, the gap between the revenue obtained through the airline's pricing strategy and the optimal revenue. Accordingly, we presume the existence of a perfect decision-maker, whose decision is always optimal given the demand distribution. The uncertainty set of a general form is concretized into differently structured uncertainty sets, which represent different information availability scenarios. We reformulate the non-convex nominal problem into a tractable form based on the available information. The closed-form solution of the reformulated model is verified as being linearly structured. We also design an efficient algorithm to characterize robust pricing strategies in polynomial-sized running times. Using numerical experiments, we investigate variations in various outputs (regret value, revenue, sales 
volume, and running time) with demand uncertainty under different information availability scenarios. The algorithm leads to robust outcomes against the demand uncertainty in practically acceptable running times, which will not increase significantly with the introduction of additional information.

The DRSP method presented in this paper is established in the robust optimization paradigm. Robust optimization is probably the most rational alternative to stochastic programming when the parameter uncertainty is not stochastic, or the probability distribution is not available (Bertsimas et al. 2011). In stochastic models, the uncertainty is assumed to have a probabilistic description (Perakis and Roels 2008). However, it is non-trivial in the air cargo industry since the cargo demand is virtually impossible to characterize in a probabilistic sense. The set-based uncertainty modeled by robust optimization is the most appropriate description of uncertainty in this context. In this paper, the robust version is formulated under a befitting decision rule, the minimax regret criterion, which minimizes the maximum possible revenue gap resulting from not making the best alternative decision (Aissi et al. 2007). Compared with the idiomatic attempt to maximize the overall profit, the minimax regret criterion is less conservative and can provide the same robustness guarantee (Jiang et al. 2013). In addition, the anticipation of the worst case over all possible scenarios is crucial in many practical applications (Aissi et al. 2009). Therefore, the minimax regret criterion is applied in a broad spectrum of real-world domains, including finance, supply chain management, statistics, and engineering, to address issues including portfolio optimization (Xidonas et al. 2017; Caçador et al. 2020; Mohammadi and Mohammadi 2018; Li and Wang 2020; Giove et al. 2006), inventory control (Yue et al. 2006; Perakis and Roels 2008, 2010; Ni et al. 2019; Campos et al. 2019), learning and estimation (Bartók et al. 2014; Alaiz-RodrÃguez et al. 2007; Eldar and Merhav 2004), behavior prediction (Jiang et al. 2013; Hayashi 2008; Kazakçi et al. 2007), and power control (Chen et al. 2014; Jo and Joo 2015; Dong et al. 2011; Li et al. 2018). Among which a typical example is the relative robust portfolio proposed by Caçador et al. (2020). The portfolio is selected from a relative robustness perspective under the minimax regret criterion, where the regret is defined as the investor's utility loss resulting from not choosing the best alternative portfolio. The real benefit of the relative robust portfolio is evaluated under the dataset with 25 -year daily historical data regarding the stocks of the DAX index. The relative robust portfolio stands out as a valid selection for risk-taking investors since it consistently presents low risks and high stability. Given the broad modeling power, our study is among the first attempts to examine the robust version of air cargo pricing from the minimax regret perspective.

The contributions of this study are fourfold. First, we investigate the uncertain contexts wherein the probability distribution is not readily available, or the parameter uncertainty is not stochastic, which has considerable practical significance. Second, this paper contributes to the literature on robust optimization by emphasizing how to preserve the model's tractability and how to immunize solutions against excessive conservatism. Third, we propose an efficient algorithm, which is imbued with tractability and high performance in the context of probabilistically indescribable uncertainties. Fourth, the findings of this study provide airlines with guidance on how to strategically collect demand information and propose prices for their capacities in different market situations.

The remainder of the paper is structured as follows. Section 2 presents a review of the relevant literature. In Sect. 3, we introduce our model and undertake model structure analysis based on different information acquisition scenarios in relation to random market demand. Section 4 presents a polynomial-sized algorithm to facilitate problem-solving, while Sect. 5 verifies the universality and robustness of the algorithm concerning distinctive underlying distributions and wider fluctuations in demand under various contexts of 
partial information acquisition. Section 6 concludes. Major proofs are provided in "Appen$\operatorname{dix} 2 "$.

\section{Literature review}

Our study is related to four streams of literature: air cargo revenue management, robust dynamic pricing, the minimax regret criterion, and incomplete information. In this section, we presented a review of the related literature from these streams and discuss how the contributions of our study differ from the existing literature.

Air cargo revenue management is a significant research field covering a wide range of topics, including network capacity planning and allocation, pricing, overbooking, rejector-accept policies, and capacity contracting. Kasilingam (1997a, b) presented an exhaustive review of the literature on air cargo revenue management, summarizing the differences between air cargo revenue management and passenger yield management. Kasilingam (1997a) developed a stochastic programming model to calculate the optimal overbooking level for discretely or continuously distributed capacity, considering factors such as spoilage costs and oversale costs. Wang and Kao (2008) examined the fuzzy-reasoning-based overbooking level in a fuzziness environment. Popescu et al. (2006) estimated the showup rate for air cargo booking using an alternative nonparametric statistical forecasting tool. The superiority of the alternative estimator over traditional estimators was verified using real-world data. Qin et al. (2012) formulated dynamic space inventory control with overbooking as a dynamic programming problem and identified constructive structural properties.

The accept-or-reject policy concerning freight forwarders' booking requests has also attracted considerable attention. Amaruchkul et al. (2007) formulated the single-flight cargo booking problem as a Markov decision process. A close-to-optimal solution was achieved using de-coupling heuristics, which offers the best performance among the various heuristics developed to deal with the high-dimensional state space of this formulation. The single-flight Markovian model was extended by Amaruchkul and Lorchirachoonkul (2011) to a multiple-flights scenario using a dynamic programming model. Amaruchkul et al. (2007) was also extended by Han et al. (2010), who examined capacity allocation in the single-leg air cargo scenario with further consideration of the cargo's profit rate. While also exploring capacity planning, Levin et al. (2012) adopted a different approach by providing an upper bound on optimal revenue with a value function approximation. Mediumto long-term contract problems have also been extensively examined in air cargo revenue management. Gupta (2008) formulated the carrier-forwarder incomplete contract problem as a Stackelberg game under the assumption of deterministic demand, which is determined by the forwarder's effort level. Amaruchkul et al. (2011) adopted a principal-agent framework to design a capacity contract between carriers and forwarders, in which allotments, lump-sum payments, and refund rates are taken into consideration and demand is assumed to be random. Moreover, Hellermann et al. (2013) proposed an options contract based on the overbooking level and reservation number to analyze the impact of overbooking on airlines' revenue.

In the abovementioned studies, the air cargo revenue problem is mostly formulated as a stochastic programming problem. Departing from these studies, we formulate it as a robust optimization problem, which is a useful method when the probability distribution is not readily available, or the parameter uncertainty is not stochastic. Our study fills the gap 
between the exact distribution required by stochastic programming methods and the realworld situation of partial information faced by the air cargo industry.

Our study is also related to the research stream of robust dynamic pricing. Lim and Shanthikumar (2007) investigated the single-product dynamic revenue management problem based on robust control when arrival rates are uncertain. They formulated the robust pricing problem as a stochastic differential game and represented model uncertainty using the notion of relative entropy. They characterized the closed-form solution through a version of Isaacs's ordinary differential equation and identified the interchangeability of the max and min, as well as the equivalence between the robust pricing problem and the exponential utility function. Wang and Xiao (2017) also investigated the dynamic price control problem and formulated the issue as a continuous-time robust-control model, albeit under the criterion of minimizing worst-case absolute regret within a given discrete price set. The distribution of the demand rate for each price point is assumed to be supported on a finite set with only the upper and lower bounds available. Recursive procedures for obtaining the optimal control policy and its superiority over classical revenue management models are also presented. Chen and Farias (2018) delineated a dynamic pricing problem for a single product over two-period selling seasons using robust optimization. They assumed that only distribution intervals of uncertain variables are perceptible, and no other information is available. The model framework they developed was accompanied by a novel algorithm, whereby the outputs were compared with those of a static pricing model. Instead of focusing on a single product, Lim et al. (2008) examined the pricing problem for multiple products with varying degrees of ambiguity. To enable coordinated resource utilization, the problem was reformulated as an equivalent risk-averse dynamic pricing problem where certainty regarding the product's revenue flow was maximized. Conversely, in the case of independent demand and resources, the problem was decomposed into risk-averse single-product problems in which certainty represented by an exponential utility function was maximized. Maximizing the worst-case profit is probably the most common pricing scheme for practical revenue management considerations. One key difference of our study from this line of research is that we are among the first attempts to choose an alternative decision rule. We minimize the expected regret value under the worst-case demand distribution. While also exploring the worst-case scenario, our study differs in focus by examining the expected loss caused by not making the best alternative decision, as presented by Wang and Xiao (2017). The alternative criterion is more in line with the pricing issues of the air freight industry than other criteria.

The minimax regret criterion has been widely discussed in the literature. The minimax regret approach was introduced by Wald (1950) and formally proposed by Savage (1972). Axiomatizations of regret decisions can be traced back to the work of Milnor (1951), who proposed a decision-theoretic axiomatization of regret. Hayashi (2008) subsequently presented the axiomatization for minimax regret for a subset of priors. Moreover, Stoye (2005) put forward axioms as necessary conditions for interim regret. Another building block of research on the minimax regret approach centers on its effect in avoiding excessive conservatism. Razin (1976) presented persuasive empirical evidence supporting the applicability of the minimax regret criterion in predicting decision-making behavior. The criterion is better at preventing excessive conservatism of the optimal outcome than another extensively used criterion, the maximin. Our study is among the first attempts to offer control over conservatism in air cargo revenue management. Given these cornerstones, the minimax regret approach has been extensively applied in multiple fields, including inventory management, revenue management, and portfolio investment. For example, Perakis and Roels (2008) used the minimax regret approach to examine the newsvendor problem with 
partial knowledge of the demand probability distribution. Order quantities were derived to minimize the maximum regret of a newsvendor for not taking the best action. Besbes and Zeevi (2009) investigated a single-product revenue management problem where prices are dynamically adjusted over a finite sales horizon. In their study, the performance of the proposed algorithms was measured using regret, which is the revenue loss relative to the maximum possible revenue with a determinate demand function. Perakis and Roels (2010) investigated the capacity allocation problem in revenue management under a robust modeling framework using both minimax and maximin regret criteria. Their results showed that, on average, minimax regret controls worked well, outperforming traditional controls over correlated or censored demand. An improvement of $2 \%$ over conventional heuristics was achieved by applying the minimax regret criterion to large real-world problem sets. More recently, Xidonas et al. (2017) incorporated future returns into the portfolio selection process by calculating the minimax regret portfolio for representative points on the efficient frontier. Their work innovatively extended the conventional minimax regret criterion to multi-objective programming problems. Taking a step forward, Caçador et al. (2020) developed a new minimax regret portfolio optimization model to investigate the out-ofsample performance of robust portfolio optimization methodologies. The authors found that, compared with the work of Xidonas et al. (2017), the proposed methodology performs more consistently by producing portfolios that perform more stably both in- and out- ofsample. From the above literature, we find that although the minimax regret criterion is an important prospect, it has not received much attention in air cargo research. To the best of our knowledge, we are among the first attempts to apply this criterion to air cargo pricing issues. In this study, the airline's anticipated regret is considered, that is, the induced inferior outcome results from not adopting the best alternative pricing decision. This study complements this stream of literature by broadening its application to include the pricing issues in the air cargo industry.

In recent years, partial visibility of uncertain information, especially demand information, has attracted increasing attention from operations management researchers, and this is also one of the considerations of our study. The demand learning approach appeals as a promising means of solving problems in this context. Wang et al. (2014) investigated nonparametric dynamic pricing problems using demand learning, wherein the regret value is minimized without predefining the functional form of the demand probability function. They developed an algorithm of lower regret compared with the algorithm proposed by Besbes and Zeevi (2009), which leads to asymptotically optimal performance as both initial inventory and demand rate increase. More recently, Malladi et al. (2018) applied a Markov chain-based framework by involving Markov-modulated demand under incomplete information regarding the demand state. They employed Bayesian updating mechanisms to incorporate the latest observed partial demand information. Qu et al. (2020) investigated sellers' adaptions to buyers' uncertain responses to the offered price in business-to-business pricing. The authors developed a Bayesian inference-based statistical learning framework to measure and update the seller's uncertainty about the demand curve as new deals take place. The benefits of this approach were verified by a case study using historical data.

Robust optimization under limited information has also been extensively studied (Perakis and Sood 2006; Yang et al. 2019; Chu et al. 2019). For example, Yang et al. (2019) examined the remanufacturing decision problem with partial random yield information. They proposed a low-cost, high-reliability robust optimization model, that is, the minimax regret approach, to derive closed-form solutions regarding effective acquisition quantities. Rather than requiring full yield information, this method only requires the support and mean of the proportional yield is required by the method, which reduces sampling cost and 
is more appropriate for real-world situations. Chu et al. (2019) developed a robust optimization framework that considers both supply and demand uncertainty, applying the framework to a multi-period, single-station inventory problem and also extending its application to multi-echelon cases. The proposed robust policies lead to cost benefits by addressing concerns on both supply and demand uncertainty. Different from the prior research, given the high costs of information collection from freight forwarders and competitors, we propose a pragmatic method for exploring optimal pricing decisions under various cases of information availability to the airline.

\section{Model}

In this section, we first introduce our proposed model and assumptions. This is followed by a discussion on how the non-convex DRSP model is reformulated to recognize the linearity of the closed-form formulae of the second-stage maximal expected regret. Proofs of all propositions are provided in "Appendix 2".

\subsection{Model setup}

We consider a setting wherein a dominant airline intends to dynamically adjust prices to sell its total capacities within a two-phase sales period in spot market of air cargo. The airline operates air cargo transport with cargo capacity $c$. The random market demand within each pricing period follows distribution $F_{i}(i=1,2)$ and is represented by stochastic price-demand function $\tilde{d}_{i}(p)(i=1,2)$. To minimize expected regret under the worst-case distribution, the airline proposes a price $p_{1}$ for its cargo capacity in the first period. Suppose there exists a perfect decision-maker, facing the same pricing problem as the airline, whose decision is always optimal given the underlying demand distribution. The ideal decisionmaker makes the first-period best alternative decision $q_{1}$ in view of the first-period demand distribution $F_{1}$. Given the first-period freight rates $p_{1}$ and $q_{1}$, the difference between the airline's revenue and the ideal decision-maker's revenue (regret value) is given by

$$
\max _{q_{1}}\left\{E_{F_{1}}\left[q_{1} \tilde{d}_{1}\left(q_{1}\right)\right]\right\}-E_{F_{1}}\left[p_{1} \tilde{d}_{1}\left(p_{1}\right)\right] .
$$

The expected regret value under the worst-case distribution is achieved by traversing across all possible distributions of $\tilde{d}_{1}\left(p_{1}\right)$

$$
\max _{F_{1}}\left\{\max _{q_{1}}\left\{E_{F_{1}}\left[q_{1} \tilde{d}_{1}\left(q_{1}\right)\right]\right\}-E_{F_{1}}\left[p_{1} \tilde{d}_{1}\left(p_{1}\right)\right]\right\} .
$$

As a result, the first period pricing problem of the airline can be written as

$$
\min _{p_{1}}\left\{\max _{F_{1}}\left\{\max _{q_{1}}\left\{E_{F_{1}}\left[q_{1} \tilde{d}_{1}\left(q_{1}\right)\right]\right\}-E_{F_{1}}\left[p_{1} \tilde{d}_{1}\left(p_{1}\right)\right]\right\}+\varphi\left(p_{1}, q_{1}\right)\right\},
$$

where $\varphi\left(p_{1}, q_{1}\right)$ is the second-period regret value given the first-period pricing decisions of the airline and the ideal decision-maker. Following the same logic, the airline offers its second-period freight rates $p_{2}$, while $q_{2}$ is the second-period price set by the ideal decisionmaker in view of the market demand distribution $F_{2}$. To minimize the maximum expected regret $\varphi\left(p_{1}, q_{1}\right)$, the airline needs to idenitfy the optimal second-period pricing strategy $p_{2}$ such that 


$$
\varphi\left(p_{1}, q_{1}\right)=\min _{p_{2}}\left\{\max _{F_{2}}\left\{\max _{q_{2}}\left\{E_{F_{2}}\left[q_{2} \min \left\{c_{q}, \tilde{d}_{2}\left(q_{2}\right)\right\}\right]\right\}-E_{F_{2}}\left[p_{2} \min \left\{c_{p}, \tilde{d}_{2}\left(p_{2}\right)\right\}\right]\right\}\right\},
$$

where $c_{p}=c-\tilde{d}_{1}\left(p_{1}\right)$ and $c_{q}=c-\tilde{d}_{1}\left(q_{1}\right)$ are remaining capacities after the realization of first-period demands.

Without loss of generality, we assume that the airline and the ideal decisionmaker make prices for their freight capacities within the same price set, that is, $p_{1}, q_{1} \in \Phi_{1}=\left\{a_{1}, a_{2}, \ldots, a_{k}\right\} ; p_{2}, \quad q_{2} \in \Phi_{2}=\left\{b_{1}, b_{2}, \ldots, b_{n}\right\}$. Moreover, we assume that demand is influenced by both price and elements of randomness. Specifically, the stochastic price-demand functions $\tilde{d}_{i}(p)$ is modeled in a multiplicative fashion:

$$
\tilde{d}_{i}(p)=\tilde{d}_{i}(p, \varepsilon)=d_{i}(p) \varepsilon,
$$

where $d_{i}(p)(i=1,2)$ is a nominal price-demand function that reflects the impact of price on demand, and the random factor $\varepsilon$ describes the impact of randomness on demand.

Further, to build a robust optimization model based on the uncertainty set that gives moments and quantiles of the random factor, we posit the following assumptions. Note that Assumptions 1-3 hold for all the following analyses, while Assumptions 4-5 relate to various cases of information availability to the airline.

Assumption 1 The random factor $\varepsilon$ lies in the interval, i.e., $\varepsilon \in[l, u]$.

Assumption 2 The $s$ th moment of $\varepsilon$ is $M_{s}, s=1,2, \ldots, n$.

Assumption 3 Nominal demand functions $d_{i}(p),(i=1,2)$ are monotonic decreasing; specifically, $d_{1}\left(a^{\prime}\right) \leq d_{1}\left(a^{\prime \prime}\right)$ for $\forall a^{\prime}, a^{\prime \prime} \in \Phi_{1}$ and $a^{\prime} \geq a^{\prime \prime} ; d_{1}\left(b^{\prime}\right) \leq d_{1}\left(b^{\prime \prime}\right)$ for $\forall b^{\prime}, b^{\prime \prime} \in \Phi_{2}$ and $b^{\prime} \geq b^{\prime \prime}$.

Assumption 4 The median of the random random factor $\varepsilon$ is $m$.

Assumption 5 The random factor $\varepsilon$ is $M_{1}$-symmetric, that is, for any $z \in\left[0, M_{1}\right], \varepsilon$ lies in the interval $\left[M_{1}-z, M_{1}\right]$ with the same probability as that of lying in the interval $\left[M_{1}, M_{1}+z\right]$.

\subsection{Model structure analysis}

The randomness of the stochastic price-demand functions $\tilde{d}_{i}\left(p_{1}\right)(i=1,2)$ is related to the random factor $\varepsilon$. Therefore, within the uncertainty set $\Psi$ that gives moments of the random factor, Eq. (4) can be further reformulated as

$$
\min _{p_{2}}\left\{\max _{F \in \Psi}\left\{\max _{q_{2}}\left\{E_{F}\left[q_{2} \min \left\{c_{q}, d\left(q_{2}\right) \varepsilon\right\}\right]\right\}-E_{F}\left[p_{2} \min \left\{c_{p}, d\left(p_{2}\right) \varepsilon\right\}\right]\right\}\right\},
$$

where

$$
\Psi=\left\{\begin{array}{l}
\int_{l}^{u} d F=1 \\
\int_{l}^{u} \varepsilon^{s} d F=M_{s}, \quad s=1,2, \ldots, n \\
d F \geq 0
\end{array}\right\}
$$


Equations (6)-(7) aim to minimize the second-stage expected regret under the worstcase distribution within the feasible distribution set $\Psi$ that specifies the moments of the random factor $\varepsilon$. To derive a more feasible form of Eqs. (6)-(7), we first consider the internal programming of the Eq. (6):

$$
\max _{F \in \Psi}\left\{E_{F}\left[q_{2} \min \left\{c_{q}, d\left(q_{2}\right) \varepsilon\right\}-p_{2} \min \left\{c_{p}, d\left(p_{2}\right) \varepsilon\right\}\right]\right\} .
$$

The following proposition gives the dual form of the Eq. (8) based on the strong duality theorem.

Proposition 1 Given $p_{2}, q_{2}$ and $c_{p}, c_{q}$, the Eq. (8) is equivalent to.

$$
\begin{aligned}
& \underset{y}{\operatorname{minimize}} y_{0}+\sum_{s=1}^{N} y_{s} M_{s} \\
& \text { subject to } y_{0}+\sum_{s=1}^{N} y_{s} z^{s} \geq q_{2} \min \left\{c_{q}, d\left(q_{2}\right) \varepsilon\right\}-p_{2} \min \left\{c_{p}, d\left(p_{2}\right) \varepsilon\right\}, \quad z \in[l, u] .
\end{aligned}
$$

Proof The strong duality theorem directly yields the above dual form of the primary model (8).

Equation (9) represents semi-infinite programming. Bertsimas and Popescu (2005) proposed solutions to semi-infinite programming by establishing an equivalent semi-programming. In the following subsections, we only consider the situations in which the moments of the random factor are known up to order zero or order one, that is, $n=0,1$. The closedform solution of problem (9) is based on various type of information about the random factor, namely the upper and lower bounds $(n=0)$, the upper and lower bounds + quantile $(n=0)$, and the upper and lower bounds + the first moment $(n=1)$. Next, we show that, given $p_{2}$ and $q_{2}$, the closed-form formulae for the second-stage maximum expected regret is well-structured.

\subsubsection{Pricing model with the distribution interval information}

The closed-form solution to problem (9) is first derived when only the upper and lower bounds of the random factor are known to the airline. Since the residual capacity pair $\left(c_{p}, c_{q}\right)$ can be any point within the two-dimensional space $[0, c] \times[0, c]$, we divide the space $[0, c] \times[0, c]$ into small domains by inserting the point set $\left\{d_{2}\left(b_{i}\right) l, d_{2}\left(b_{i}\right) u, i=1,2, \ldots, n\right\}$ into the horizontal and vertical intervals $[0, c]$. Therefore, the two-dimensional space $[0, c] \times[0, c]$ is divided into $T=(2 n+1)^{2}$ small domains. We denote each small domain as $\Delta t, t=1,2, \ldots, T$. The following discussion is based on this division. Proposition 2 illustrates the modality of the closed-form solution to the problem (9) within each domain $\Delta t$.

Proposition 2 Given $p_{2}$ and $q_{2}$, for positive random factor $\varepsilon$, supposing that Assumption 1 holds and is visible to the airline, the closed-form solution to the problem (9) in each small domain $\Delta t$ is a linear or piecewise linear function of $c_{p}$ and $c_{q}$. The closed-form formulae are shown in Table 1 in "Appendix 1".

Given the upper and lower bounds of the random factor, Proposition 2 yields the closedform formula for the optimal solution to the problem (9). Note that the linear or piecewise linear form of the optimal solution also holds for robust optimization form or stochastic 
form (Chen and Farias 2018). Next, we examine the modality of the optimal solution when the airline can obtain additional quantile information regarding the random factor.

\subsubsection{Pricing model with the distribution interval and quantile information}

Although it is difficult for the airline to obtain complete demand information, and thus estimate the exact demand distribution, quantile information is deemed to be fully helpful in enabling the airline to enhance its freight management stability and increase revenue. As a result, an airline would carefully collect information on quantiles of demand and use boxplots to capture the underlying demand distribution. This inspired us to solve the optimization problem using quantile information regarding the random factor.

We derive the structural properties of the optimal solution to the problem (9) based on the distribution interval and the median given by Assumptions 1 and 4. It is worth mentioning that although the structural properties yielded by Proposition 3 are based on the median of random factor $\varepsilon$, the properties can be naturally generalized to other quantiles, such as the 25th and 75th percentiles.

Similar to the treatment of the two-dimensional space $[0, c] \times[0, c]$ in Sect. 3.2.1, we insert the point set $\left\{d_{2}\left(b_{i}\right) l, d_{2}\left(b_{i}\right) u, d_{2}\left(b_{i}\right) m, i=1,2, \ldots, n\right\}$ into the horizontal and vertiacal intervals $[0, c]$ to obtain at most $(3 n+1)$ subintervals in each dimension. Therefore, the two-dimensional space $[0, c] \times[0, c]$ is divided into $T=(3 n+1)^{2}$ small domains. For ease of record, we continue to use $\Delta t, t=1,2, \ldots, T$ to denote the small domains. Proposition 3 yields the structural property of the optimal solution to the problem (9) within each small domain in the presence of distribution interval and quantile information.

Proposition 3 Given $p_{2}$ and $q_{2}$, for positive random factor $\varepsilon$, suppose that Assumptions 1 and 4 hold and are visible to the airline. The closed-form solution to the problem (9) in each small domain $\Delta t$ is a linear or piecewise linear function of $c_{p}$ and $c_{q}$. The closed-form formulae are shown in Table 2 in "Appendix 1".

When the two-dimensional capacity space is divided into smaller domains based on the information obtained, the linearity of the closed-form solution always holds within each small domain. The linear structural property simplifies the pricing problem and facilitates the subsequent algorithm design.

\subsubsection{Pricing model with zero-order and first-order moment information}

In the previous two subsections, we assumed that the moments of the random factor are knowable up to order zero $(n=0)$, that is, only the upper and lower bounds of the random factor are accessible to the airline. Next, we show that the closed-form solutions to the problem (9) are still linear or piecewise linear functions of $c_{p}$ and $c_{q}$ under the additional consideration of the first-order moment of the symmetrically distributed random factor $\varepsilon$.

For ease of implementation, we make a approximate symmetry-based treatment for the random factor $\varepsilon$. Under Assumption 5, it can be seen that $\mu$-symmetric random factor $\varepsilon$ has a median value of $\mu(=m)$. We divide the two-dimensional space $[0, c] \times[0, c]$ into at most $T=(2 n+1)^{2}$ small domains by inserting the set of points $\left\{d_{2}\left(b_{i}\right) m, 2 d_{2}\left(b_{i}\right) m, i=1,2, \ldots, n\right\}$ into the horizontal and vertical intervals $[0, c]$. The small domains are again denoted as $\Delta t, t=1,2, \ldots, T$. Proposition 4 yields that the linearity of the optimal pricing decision still holds when the moments of a symmetrically distributed random factor are attainable up to 
order one, that is, in addition to the distribution interval, the mean value information is also visible to the airline.

Proposition 4 Given $p_{2}$ and $q_{2}$, for positive random factor $\varepsilon$, suppose that Assumptions 1, 2 and 5 hold and are visible to the airline. The closed-form solution to the problem (9) in each small domain $\Delta t$ is a linear or piecewise linear function of $c_{p}$ and $c_{q}$. The closed-form formulae are shown in Table 3 in "Appendix 1".

Under this division of the capacity space $[0, c] \times[0, c]$, consideration of the first-order moment does not disturb the linear structure of the closed-form solution within each small domain. The analysis is based on an asymptotic treatment, which assumes that the random factor is symmetrically distributed over the distribution interval. This approximate symmetrybased treatment preserves the structural properties and facilitates the solving process. However, the asymptotic treatment might not be applicable to those random variables with asymmetric distribution because, in this case, it may lead to information wastage and reduce the value of the mean information.

\section{Polynomial algorithm design}

In the previous section, by dividing the two-dimensional capacity space into small domains based on perceived information, we showed that the closed-form solution to the problem (9) is a linear or piecewise linear function of $c_{p}$ and $c_{q}$ within each domain. Now we proceed to the model

$$
\max _{q_{2}}\left\{\max _{F \in \Psi}\left\{E_{F}\left[q_{2} \min \left\{c_{q}, d_{2}\left(q_{2}\right) \varepsilon\right\}-\left[p_{2} \min \left\{c_{p}, d_{2}\left(p_{2}\right) \varepsilon\right\}\right]\right\}\right\} .\right.
$$

The model (10) solves for the maximum of a group of functions of size $O(T)$ within each domain. Therefore, the model (10) is a convex piecewise linear function of $c_{p}$ and $c_{q}$ with at most $O(T)$ piecewise intervals. Accordingly, the model (6) solves for the minimum of a set of convex piecewise linear functions. For ease of implementation, we further divide each small domain $\Delta t$ into at most $O\left(T^{2}\right)$ subdomains and denote these subdomains as $\delta_{h}, h=1,2, \ldots, H$, where $H=O\left(n^{4}\right)$. Proposition 5 indicates the linearity of the secondstage regret $\varphi\left(p_{1}, q_{1}\right)$ within each small subdomain.

Proposition 5 In each subdomain $\delta_{h}, h=1,2, \ldots, H, \varphi\left(p_{1}, q_{1}\right)$ is a linear function of $\tilde{d}_{1}\left(p_{1}\right)$ and $\tilde{d}_{1}\left(q_{1}\right)$.

We denote the linear function in Proposition 5 as $l\left(\tilde{d}_{1}\left(p_{1}\right), \tilde{d}_{1}\left(q_{1}\right)\right)$. The constraint of each subdomain can also be linearly represented in terms of $\tilde{d}_{1}\left(p_{1}\right)$ and $\tilde{d}_{1}\left(q_{1}\right)$ as $C\left(\tilde{d}_{1}\left(p_{1}\right), \tilde{d}_{1}\left(q_{1}\right)\right) \leq 0$. Accordingly, the internal programming of the model (3) can be written as:

$$
\begin{array}{cl}
\underset{\bar{d}_{1}\left(p_{1}\right) \bar{d}_{1}\left(q_{1}\right)}{\operatorname{maximize}} & \left\{q_{1} \bar{d}_{1}\left(q_{1}\right)-p_{1} \bar{d}_{1},\left(p_{1}\right)\right\}+\varphi\left(p_{1}, q_{1}\right) \\
\text { subject to } & l_{1}\left(p_{1}\right) \leq d_{1}\left(p_{1}\right) \leq u_{1}\left(p_{1}\right), \\
& l_{1}\left(q_{1}\right) \leq d_{1}\left(q_{1}\right) \leq u_{1}\left(q_{1}\right), \\
& \left(d_{1}\left(p_{1}\right)-d_{1}\left(q_{1}\right)\right)\left(p_{1}-q_{1}\right) \leq 0, \\
& C\left(\tilde{d}_{1}\left(p_{1}\right), \tilde{d}_{1}\left(q_{1}\right)\right) \leq 0 .
\end{array}
$$


Note that $\bar{d}_{1}\left(p_{1}\right)$ and $\bar{d}_{1}\left(q_{1}\right)$ represents expected demand given prices $p_{1}$ and $q_{1}$. Therefore, maximizing the objective function in the model (11) is equivalent to maximizing the deterministic parts $d_{1}\left(p_{1}\right)$ and $d_{1}\left(q_{1}\right)$. For each available price pair $\left(p_{1}, q_{1}\right) \in \Psi_{1} \times \Psi_{1}$, the optimal pricing decision is obtained when the terms involving $p_{1}$ are minimized and those involving $q_{1}$ are maximized. Traversing all the sub-domains yields the optimal $\delta_{h}$ that provides the optimal pricing decision $\left(p_{2}, q_{2}\right)$ of the second period. Theorem 1 guarantees a running time of order $O\left(k^{2} n^{4}\right)$ for the proposed algorithm.

Theorem 1 The optimum value for the model (3) can be achieved within time $O\left(k^{2} n^{4}\right)$.

Proof The linear or piecewise linear structural property yielded by Propositions 2-5 ensures that the model (11) is solved within time $O(1)$ (Chen and Farias 2018). As a result, $O\left(n^{4}\right)$ subdomains and $k^{2}$ price pairs $\left(p_{1}, q_{1}\right)$ lead to a running time of $O\left(k^{2} n^{4}\right)$.

\section{Numerical experiments}

In this section, we will verify the efficiency and generalizability of the proposed algorithm. We conduct numerical experiments under various demand information contexts and differently distributed random factors. We presume that the random factor follows one of five distributions (uniform, normal, gamma, beta, or log-normal distribution). By fine-tuning the distribution parameters, the optimal pricing decision, regret value, average revenue, sales volume, and the running time are achieved under differently distributed random factors. To enable a comparison between the performance of the proposed algorithm and that of the algorithm put forward by Chen and Farias (2018), the data set used by Chen and Farias (2018) is used in this study.

\subsection{Input parameters}

We consider the discrete price sets $\Psi_{1}=\Psi_{2}=\{50,55,60,66,72,79,86,94,103,113\}$. The nominal demand function is given by $d(p)=50 \times(p / 113)^{-1.5}$ in both sales periods. The airline has a total capacity of $c=2 d\left(p_{m}\right)$, where $p_{m}$ is the median of feasible prices given by the discrete price sets $\Psi_{1}$ and $\Psi_{2}$. Suppose the random factor has a lower bound $l=1-v$ and an upper bound $u=1+v$, where $v \in[0.05,0.5]$.

In the first set of experiments, the random factor $\varepsilon$ is drawn from a normal truncation distribution with mean value $M_{1}=(l+u) / 2$ and standard deviation $\sigma=(u-l) / 4$. In the second set of experiments, the random factor $\varepsilon$ is presumed to be uniformly distributed on support $[l, u]$. In the third set of experiments, the random factor is gamma-distributed on support $[l, u]$ with a shape parameter of 2 and a scalar parameter of 0.5 . In the fourth set of experiments, the random factor $\varepsilon$ follows a beta distribution with both scalar parameters of 0.5. The fifth set of experiments consider log-normal distribution, wherein the logarithms of $\mu$ and $\sigma$ are set as 0 and 0.5 , respectively.

Under the various distributions of the random factor, the optimal pricing decisions are obtained under three information availability scenarios: upper and lower bounds, upper and lower bounds + median, and upper and lower bounds + mean value. 


\subsection{Results and implications}

We investigate variations in the outputs of interest (regret value, revenue, sales volume, and running time) with demand uncertainty under different information premises (upper and lower bounds, upper and lower bounds + median, and upper and lower bounds + mean value). The level of demand uncertainty is measured by the length of the semi-distribution interval of the random factor, which reflects the effect of randomness on demand. The feasibility and universality of the algorithm are verified under the various distribution types (normal, uniform, beta, and log-normal distribution).

\subsubsection{Regret value}

We plot the regret value with respect to the length of the semi-distribution interval under various distributions of the random factor (see Fig. 1). It reveals how the regret value resulting from the pricing strategy might respond to the demand volatility under various types of information. We choose absolute regret, which measures the performance of the algorithm relative to the ideal decision-maker, as the objective value of the proposed algorithm. Another common metric is relative regret, which is the ratio of absolute regret to the optimal revenue. We only present absolute regret because absolute regret and relative regret follow similar trends, both increasing as the length of the interval increases. This is attributed to the fact that average revenue decreases as demand uncertainty intensifies (see Fig. 2).

The regret is insensitive to the length of the distribution interval when the airline is aware of the upper and lower bounds and the mean value, regardless of the distribution of the random factor. This insensitivity can also be seen when examining the variations in revenue and sales volume with variations in demand uncertainty (See Figs. 2, 3). The insensitivity lies in the fact that the lower bound $(1-v)$ and the upper bound $(1+v)$ are symmetric. Take the normal distribution as an example. Given the lower bound $(1-v)$, the upper bound $(1+v)$, and the distribution function $F$, the median (which is also the mean for a symmetric distribution) of the truncated normal distribution is given by $F^{-1}\left(\frac{1}{2} F(1-v)+\frac{1}{2} F(1+v)\right)$. If the random factor is symmetrically distributed, the median is constant regardless of the value of $v$. For asymmetric random distributions such as the beta distribution, the change in the median or the mean is also extremely small. As shown in Sect. 3.2.3, the stability of the median or the mean makes the algorithm outputs relatively stable.

We find that the regret value of the optimal pricing decision, which considers the upper and lower bounds and the median, is always smaller than the regret value when only information regarding the upper and lower bounds is available. Moreover, the gap increases as the length of the distribution interval increases, except when the random factor follows a beta distribution. That is to say, as demand uncertainty intensifies, the value of median information in reducing the regret value is magnified. Therefore, the airline should pay close attention to the median information, especially in an unstable market environment.

We also find that whether information on the mean value contributes to regret reduction depends on the level of demand uncertainty. Extra access to mean value information reduces the regret value only when the distribution interval is sufficiently long. In other words, only when the demand fluctuates significantly can mean value information help to reduce regret. Therefore, the mean value should be overlooked when the market is relatively stable but needs to be closely observed when the demand uncertainty increases. 


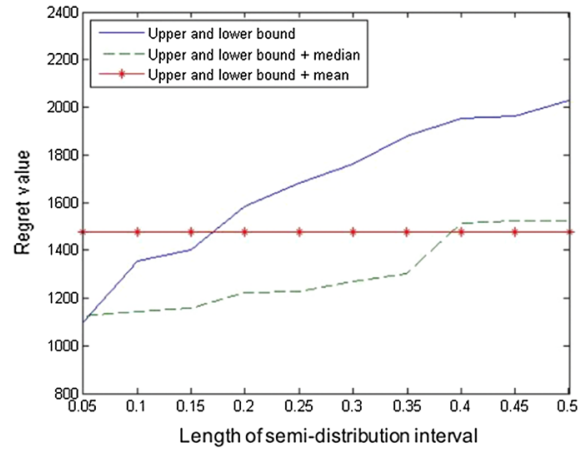

(a) Normally distributed random factor

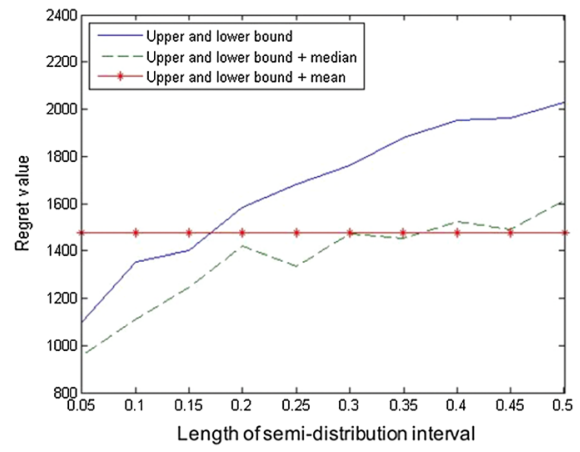

(c) Gamma-distributed random factor

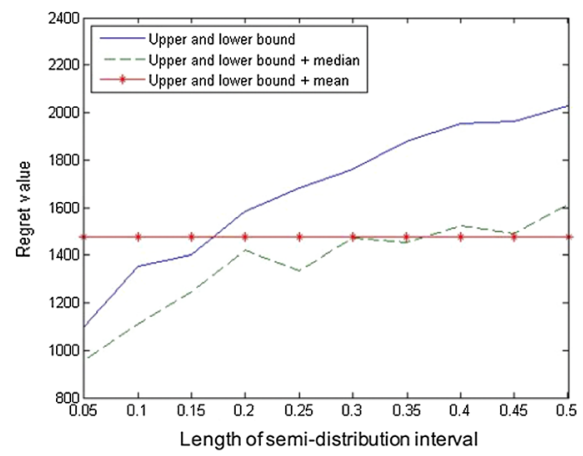

(e) Log-normally distributed random factor

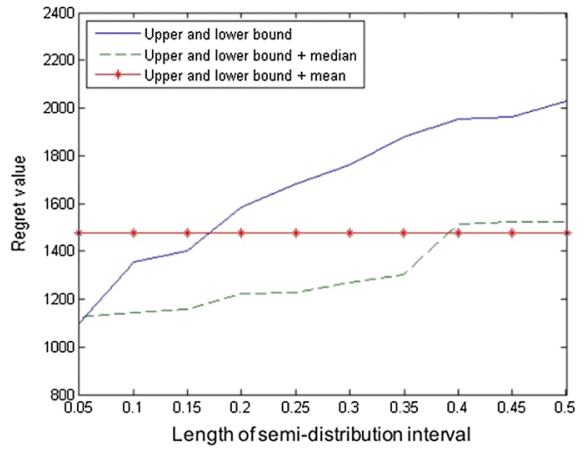

(b) Uniformly distributed random factor

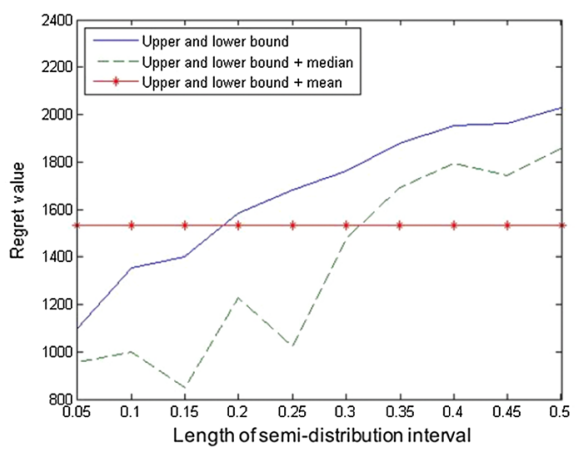

(d) Beta-distributed random factor

Fig. 1 Regret value under different demand information

\subsubsection{Average revenue}

To investigate the performance of the average revenue in response to demand uncertainty under various information scenarios, in Fig. 2, we plot the regret value with respect to the length of the semi-distribution interval under various distributions of the random factor. We find that, in most cases, average revenue shows a downward trend as the distribution interval increases. This trend can also be observed when we compare the revenue 


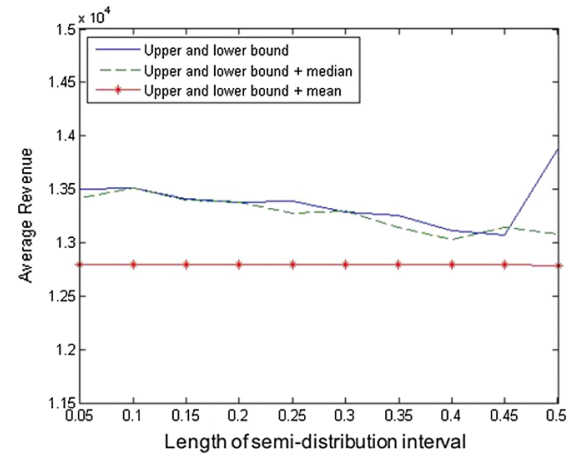

(a) Normally distributed random factor

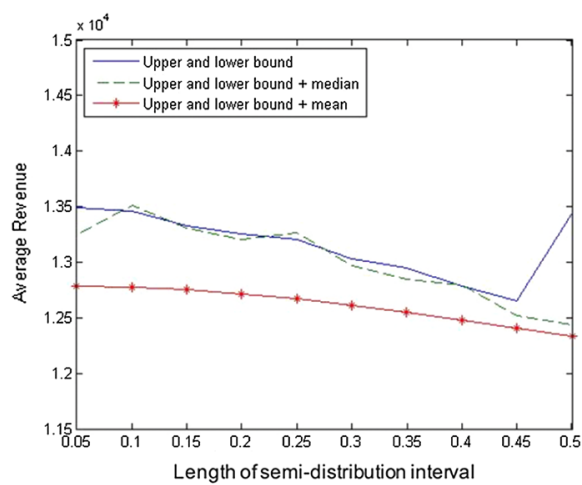

(c) Gamma-distributed random factor

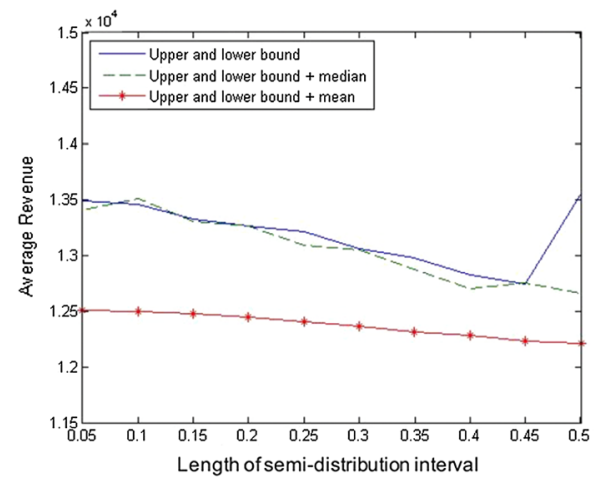

(e) Log-normally distributed random factor

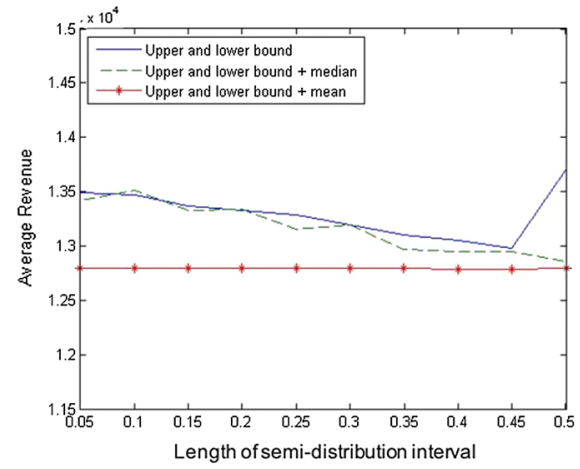

(b) Uniformly distributed random factor

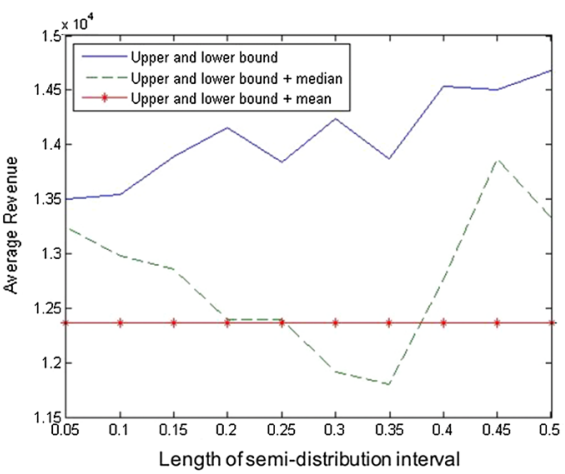

(d) Beta-distributed random factor

Fig. 2 Average revenue under different demand information

performance of the deterministic inventory model and that of the stochastic inventory model. Specifically, when the lower bound and upper bound are very close or equal, the stochastic optimization problem is reduced to a deterministic problem. Naturally, there is no sales loss because of uncertainty. However, as the length of the distribution interval increases, the increasing uncertainty results in an additional loss of sales and revenue. 


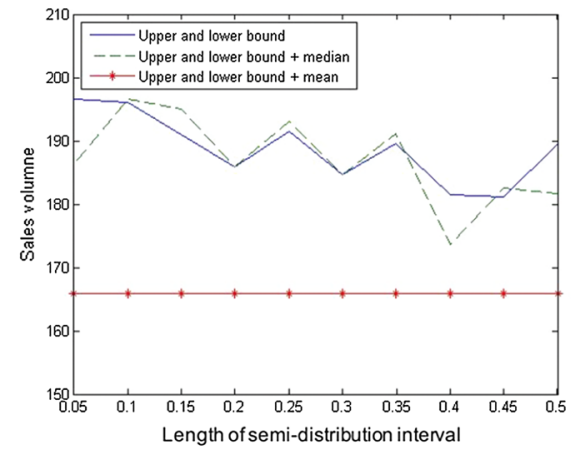

(a) Normally distributed random factor

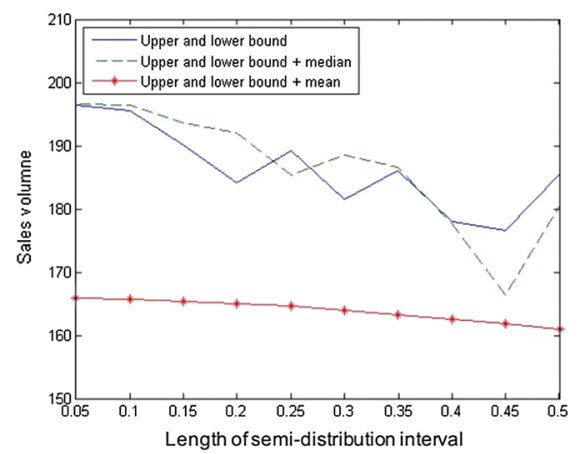

(c) Gamma-distributed random factor

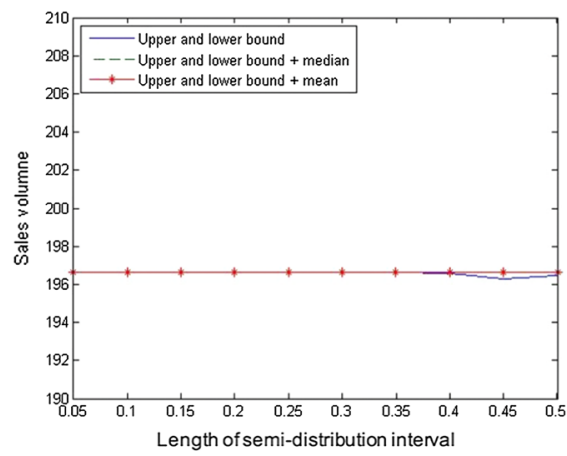

(e) Log-normally distributed random factor

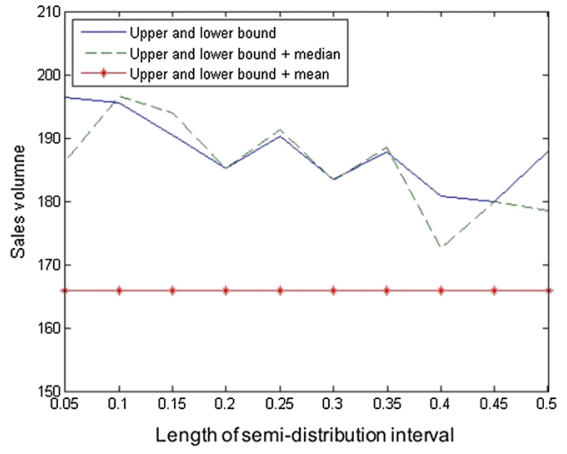

(b) Uniformly distributed random factor

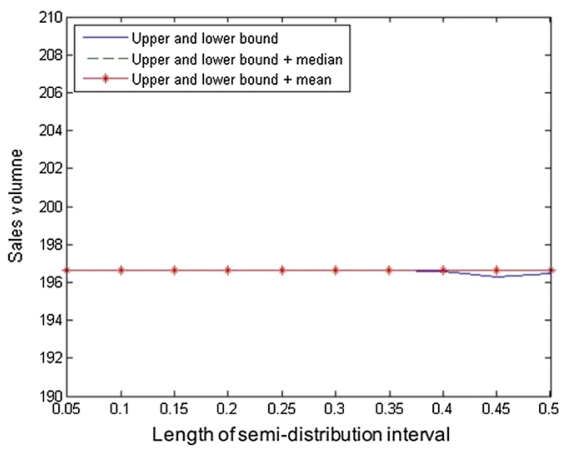

(d) Beta-distributed random factor

Fig. 3 Sales volume under different demand information

However, we believe that this reduction in revenue (within 15\%) is generally acceptable compared with the change in the distribution interval (a tenfold increase).

We also find that additional access to information on the median results in revenue that is at approximately the same level as that when only information regarding the upper and lower bounds is available. Combined with Fig. 1, we can deduce that information on the median 
reduces regret, but not at the expense of revenue. However, no increase in revenue can be seen with the addition of median information. Therefore, on the one hand, airlines need to be aware that introducing information on the median is unlikely to increase average revenue. On the other hand, they should leverage information on the median, especially when demand fluctuates significantly, to reduce regret, but not at the expense of average revenue.

Furthermore, it can be seen that output based on additional information on the mean value does not respond to demand uncertainty, regardless of the distribution type. This is attributed to the fact that optimal pricing decisions that consider the mean value are based on symmetric approximations. These approximations not only result in insensitivity but also hurt the revenue because they might lead to information loss, thereby reducing the worth of mean value information.

\subsubsection{Sales volume}

To investigate how sales volume might respond to demand volatility under various information types, in Fig. 3, we plot sales volume with respect to the length of the semi-distribution interval under various types of distribution of the random factor. We find that sales volume generally follows a similar trend to that followed by revenue. When only the zeroorder moment or both the zero-order mean and median are available to the airline, sales volume shows a fluctuating decreasing trend as demand uncertainty intensifies, except when the random factor follows a beta distribution. When mean value information is available to the airline, sales volume does not respond to variations in the distribution interval because of our approximate symmetry-based treatment.

We also find that additional access to information on the median or the mean does not lead to higher sales volumes compared with the case where only information regarding the upper and lower bounds is available. Therefore, airlines need to be aware that introducing information on the median or the mean is unlikely to increase sales volume but is likely to reduce sales.

\subsubsection{Running time}

To demonstrate the feasibility of the proposed algorithm under different information types, in Fig. 4, we plot the running time (measured in seconds) with respect to demand uncertainty under various distributions of the random factor. Almost all the experiments finished within three minutes, which is acceptable in practice. More importantly, the inclusion of additional information (such as that regarding the median or the mean value) does not incur additional costs of running time (see Fig. 4). Although the running time increases slightly when information on the median is included, the increase is insufficient to give rise to a magnitude change. Conversely, the inclusion of information on the mean value significantly shortens the running time when demand is symmetrically distributed. The running time benefits from the symmetry-based approximation, and thus a reduction only occurs in markets where demand is symmetrically distributed.

Figure 4 implies that, on the one hand, the airlines do not need to worry that additional information will reduce the feasibility of their pricing strategies. Instead, sometimes additional information makes pricing strategies easier to execute. On the other hand, the airlines need to be aware that knowledge of mean values only accelerates the execution of pricing decisions in markets with symmetric distributions. 


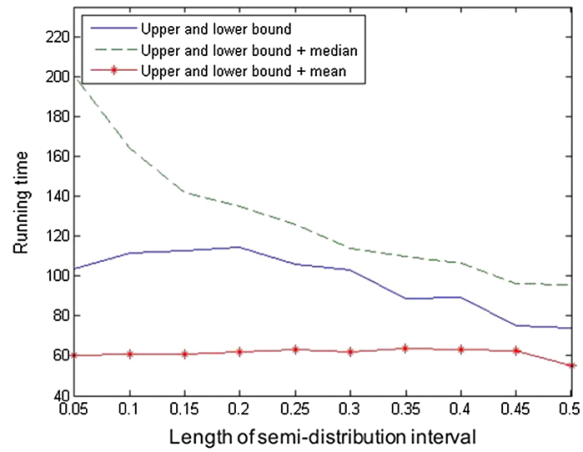

(a) Normally distributed random factor

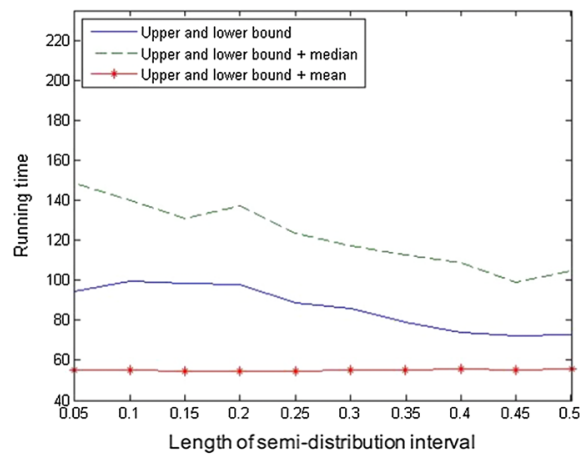

(c) Gamma-distributed random factor

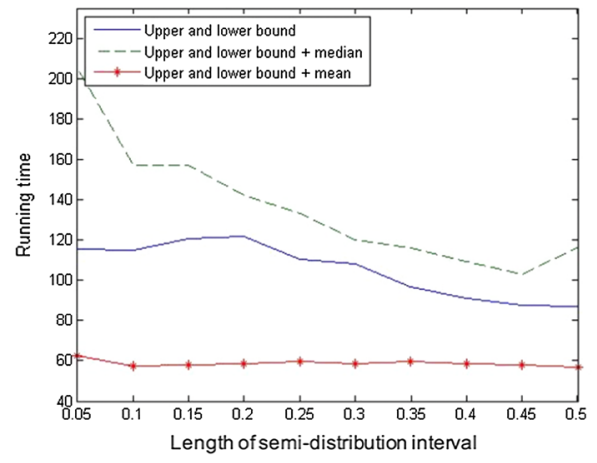

(e) Log-normally distributed random factor

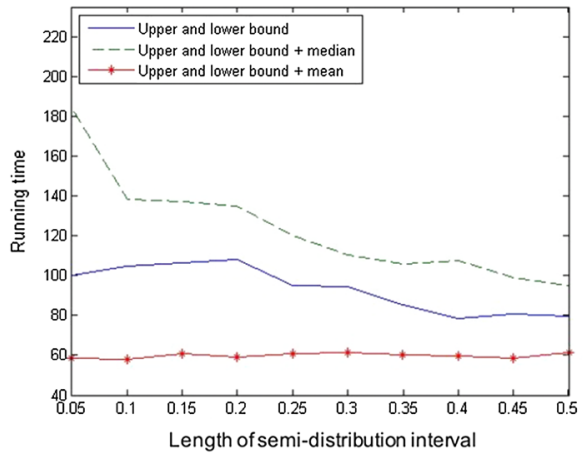

(b) Uniformly distributed random factor

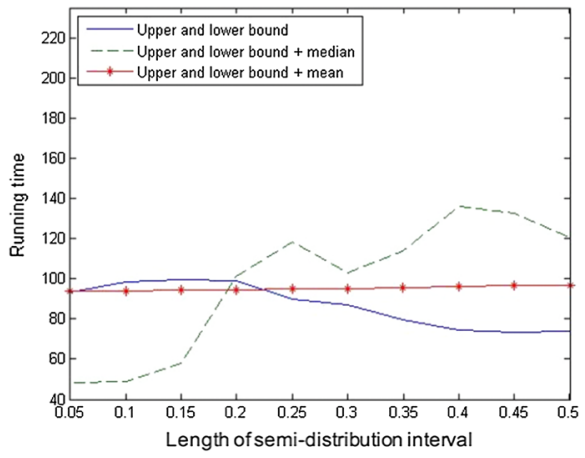

(d) Beta-distributed random factor

Fig. 4 Running time under different demand information

\section{Conclusion}

In this study, we model the problem of dynamic pricing as a DRSP with the addition of partial demand information. Instead of maximizing overall profitability, the model aims to minimize expected regret under the worst-case demand distribution. The proposed non-convex DRSP model was further reformulated based on the information obtained by the airline. The 
closed-form solution of the reformulated model was identified as either linear or piecewise linear within each small domain of the divided capacity space. The linear structural property simplifies the pricing problem and facilitates the design of a pricing algorithm. Using the proposed algorithm, we performed numerical experiments to investigate variations in key outputs (regret value, revenue, sales volume, and running time) with demand uncertainty under different information premises. The results demonstrated robustness against demand uncertainty, regardless of the underlying demand distribution. Comparison of experimental outcomes revealed the effect of additional information in various market environments. The numerical experiments' running time was verified as practically acceptable, and did not increase significantly with the introduction of additional information.

The results of this study provide several useful managerial insights. First, our findings provide guidance for airlines to strategically collect demand information based on demand fluctuations. The experimental results revealed whether the value of specific information is amplified or weakend as the market volatility intensifies. The market situation, in which specific demand information needs to be taken full advantage of by airlines, was identified. A comprehensive grasp of demand information is generally useful in facilitating pricing decisions, especially when the airline is faced with a high degree of uncertainty. As shown in Fig. 1, a dynamic pricing strategy based on additional percentile information achieves a lower level of regret compared with the strategy based only on information regarding the lower and upper bounds. This also holds when considering relative regret (the ratio of regret to revenue) because information on the median reduces regret, but not at the expense of revenue. The average revenue is roughly the same regardless of whether information on the median is included (see Fig. 2). Moreover, the reduction in regret becomes more significant as the length of the distribution interval increases. In other words, as demand uncertainty intensifies, the value of information on the median increases in terms of reducing regret. However, information on the median does not lead to significant changes in average revenue and sales volume. Therefore, on the one hand, the airlines need to be aware that including information on the median is unlikely to increase average revenue or sales volume. On the other hand, they should leverage information on the median, especially when market demand fluctuates significantly, to reduce regret, but not at the expense of sales and revenue. Access to information on the mean value reduces regret only when demand fluctuates significantly. Further, the higher the uncertainty in demand, the more significant the reduction in regret. Therefore, the mean value should be overlooked by the airlines when the market is relatively stable but needs to be included when demand uncertainty increases.

Second, we find that the loss is negligible relative to demand volatility even though increased demand uncertainty results in a decline in sales and revenue. When demand uncertainty increases tenfold, revenue declines by just $8 \%$ (except for the case of beta-distributed demand where changes in average revenue of up to $15 \%$ occurs). Generally speaking, fluctuations in demand due to overbooking, cancellations, reservation updating, and variable tenders inevitably impose additional risks on the airlines' pricing decisions. Revenue and sales are normally the first casualties of airlines' risk-taking. However, the stability of these outcomes under the DRSP modeling framework implies that the risk is largely controllable. Airlines can strategically adjust their pricing to incorporate pricing uncertainties and absorb pricing risks. These pricing decisions are immunized against demand uncertainty, and the airlines' anxiety caused by their exposure to uncertain demand and pricing risks is significantly reduced.

Third, the results of this study show that additional information does not compromise the viability of the pricing strategies being implemented. Therefore, the proposed method is easier for airlines to use. The pricing strategies based on various information types all 
result in polynomial-sized running time, which is acceptable in practice. The consideration of additional information (such as that information regarding the median or mean) does not incur high additional cost in terms of the running time (see Fig. 4). Although the running time increases slightly when the median is considered, this increase is not sufficient to give rise to a magnitude change. Instead, the addition of the mean value reduces the running time when demand is symmetrically distributed. Therefore, the inclusion of additional information will not reduce the feasibility of the pricing strategies being executed. Sometimes it might actually make them easier to execute. However, the airlines need to be aware that the addition of mean value information accelerates pricing decisions only in markets with symmetrically distributed randomness.

The key contributions of this study are fourfold. First, we investigate uncertain contexts wherein either the probability distribution is not readily available, or the parameter uncertainty is not stochastic. Many real-world problems may encounter uncertainty without a probabilistic description. Instead of considering stochastic uncertainty in some probabilistic sense, we developed a DRSP model under which robustness is sought against set-based uncertainty. A significant advantage of this method is that it does not require precise probability distributions. The method also better reconciles conservatism and performance. It imbues the solutions with robustness while immunizing them against over-conservatism. Moreover, this approach preserves computational tractability, which is usually the starting point for exploring practical feasibility. In view of these advantages, the model is expected to be used across a broad range of domains, especially those with probabilistically indescribable uncertainties. For example, the method is highly suitable for the dynamic pricing problem of the air cargo industry that is examined in this study. Air cargo demand is subject to considerable volatility, making it difficult to estimate in a probabilistic sense. This leads to great anxieties among airlines attempting to make prices for their capacities. The DRSP model empowers the airlines' pricing decisions to respond to demand uncertainty and hedge against demand risk. The model also provides operational decision-making guidance in other uncertain or imprecise contexts, such as dynamic pricing and sales promotion in the apparel and giftware industries. However, it is worth mentioning that the DRSP method proposed in this study is a general model, which needs to be slightly modified based on the diversity of capacity constraints and decision-maker preferences in different uncertain contexts.

Second, the results of this study enrich the research on robust optimization by emphasizing how to preserve the model's tractability and how to immunize solutions against excessive conservatism. Preserving tractability is a primary goal of robust models. Generally, the robust version of an optimization problem may not itself be tractable. Tractability relies on the structure of the nominal problem and the formulation of the uncertainty set. We regard these two factors as the starting point for establishing a tractability guarantee. We consider set-based uncertainty with a particular focus on the sets that describe the moment and quantile uncertainty of demand. The uncertainty set describing moments and quantiles of demand is concretized into uncertainty sets with different formations. Under these differently structured uncertainty sets, we reformulate the original nominal problem into a wellstructured form using the strong dual theorem. The reformulation is supported by a division of the capacity space in the presence of specified moment and quantile information. The closed-form solution of the reformulated model is verified as linearly structured within each small domain. The linear structural property guarantees the computational tractability of the model. In addition to the tractability guarantee, the conservativism of robust optimization is also a key issue. We immunize the solutions to excessive conservatism by choosing an alternative subjective decision rule, that is, minimizing the expected regret under the worst-case demand distribution. Instead of focusing on the worst-case profit under the 
maximin criterion, we consider the loss caused by not making the best decision. Under this alternative criterion, the solutions need not be overly conservative, but can also be immune to uncertainty. Therefore, a balance between conservatism and performance is achieved.

Third, our proposed algorithm is imbued with tractability and high performance in the context of probabilistically indescribable uncertainties. The feasibility of the algorithm is guaranteed by eliminating the uncertain components of demand and reformulating the original problem into a well-structured form. We verify that, under the division of the capacity space, in each divided domain, maximizing the objective value of the original problem amounts to solving linear programming with respect to the deterministic parts of the uncertain demand. The deterministic part of this demand is uniquely determined by the price and is unrelated to randomness. In the reformulated problem, for each feasible price pair, the optimal solution within each small domain is achieved as long as the terms concerning the airline's pricing decision are minimized, whereas the terms concerning the best alternative pricing decisions are maximized. All the small domains are examined to identify the one that yields the optimal pricing decision. In addition to providing a feasibility guarantee, the algorithm exhibits good performance. The outputs of the algorithm demonstrate robustness against demand volatility regardless of the underlying demand distribution. These robust outcomes can be achieved during a polynomial-sized running time regardless of the demand information on which the pricing decision is based. Consideration of additional information (such as the median or mean value) does not result in a significant increase in the running time. Indeed, in certain market environments, specific additional information is likely to reduce the running time. The efficiency of the proposed algorithm enables the airlines to respond promptly to the demand uncertainty.

Last, the findings of this study provide airlines with guidance in strategically collecting demand information and pricing their capacities in different market situations. The numerical results present variations in economic outcomes with demand uncertainty under various information availability scenarios. Comparison of these outcomes presents an indication of whether the value of the additional information is magnified or reduced as the market volatility increases. Thus, it is possible to identify the market situation under which specific additional demand information needs to be taken full advantage. Therefore, airlines can strategically gather demand information based on the market environment to maximize the utility obtained by collecting additional information.

We suggest several research topics for future research. First, it would be worthwhile to develop an integrated capacity planning and sales strategy formulation framework that synthesizes air freight capacity demand for both long-term contracts and spot markets in the air cargo industry. Such a framework would provide theoretical guidance for the logistics industry and service providers in terms of their capacity planning and pricing decisions; Second, it is worth investigating how the general model proposed in this study can be modified in response to diverse capacity constraints and decision-maker preferences. Third, it would be worthwhile investigating how the approximate symmetry-based treatment can be improved without causing a significant increase in complexity. These topics are particularly promising in light of the unexplored value-creation opportunities identified in the study.

\section{Appendix 1}

See Tables 1, 2 and 3. 


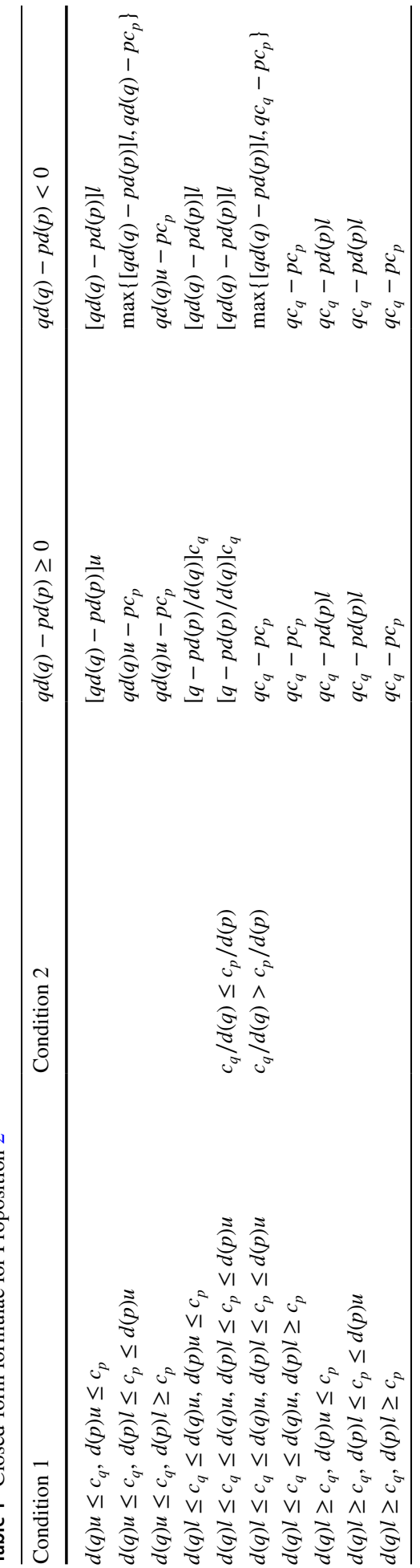



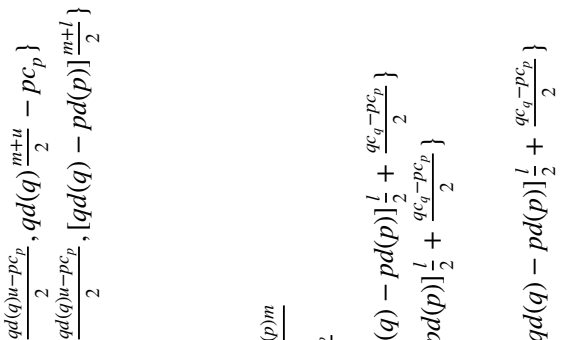

$++$

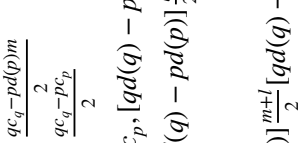

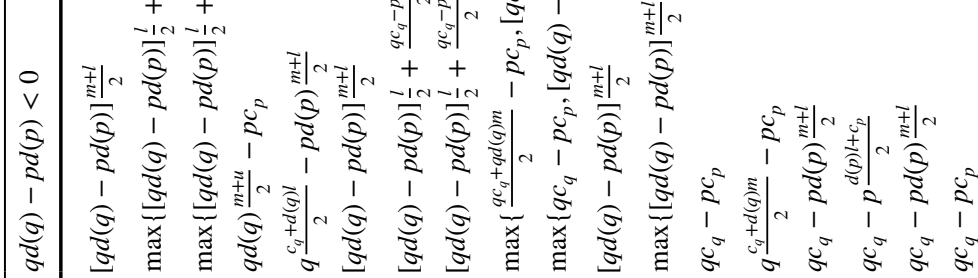

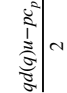

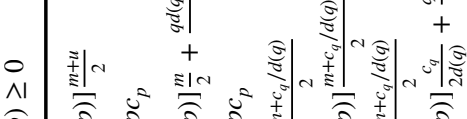

空。

(1)

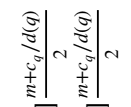

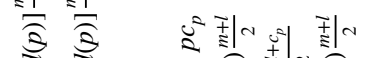

亲

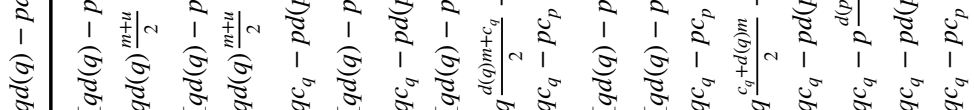

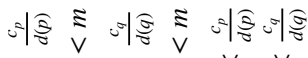

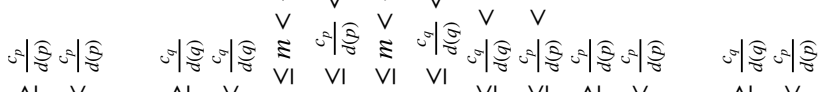

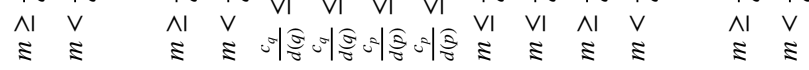

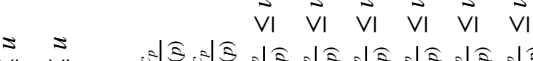

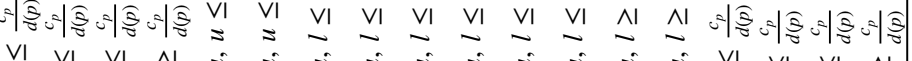

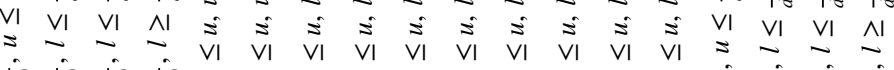

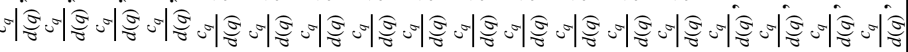

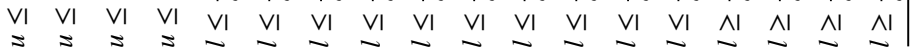




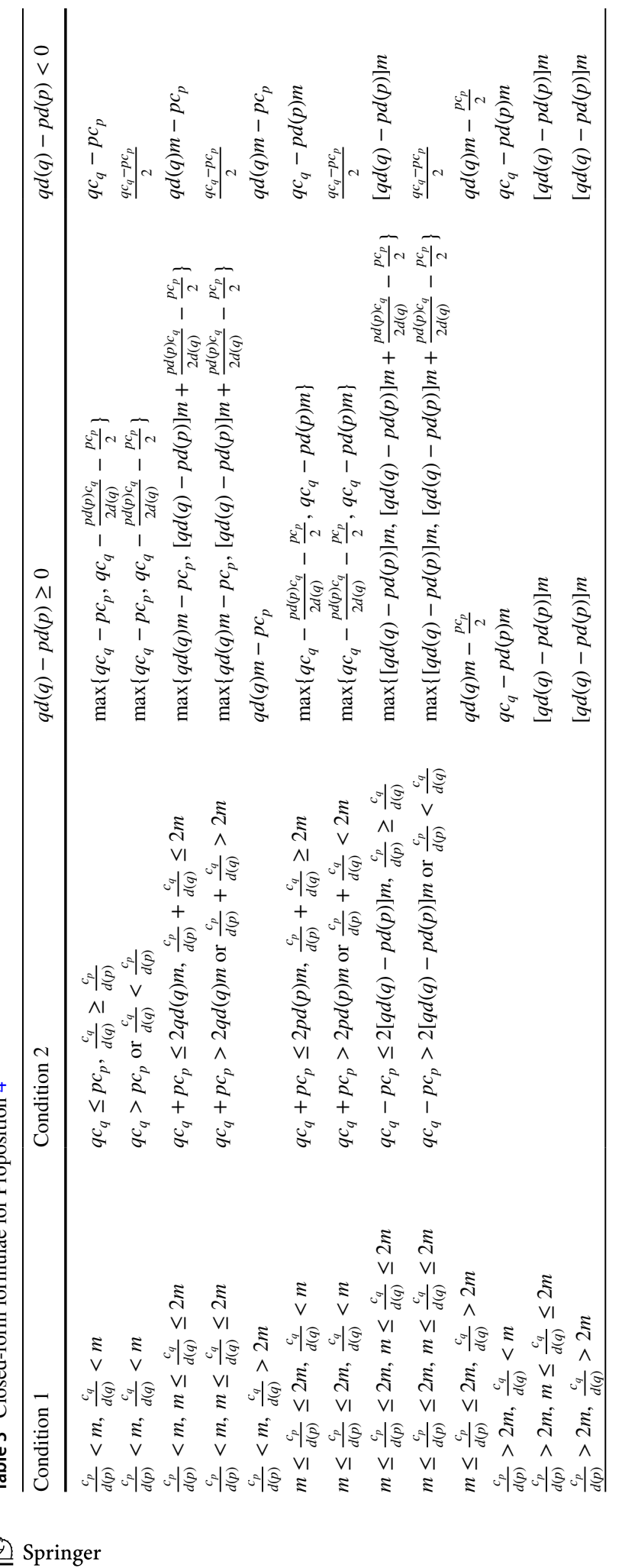




\section{Appendix 2}

Proof of Proposition 2 The optimal value of problem (9) can be formulated as:

$$
\max _{l \leq z \leq u}\left\{q \min \left\{c_{q}, d(q) z\right\}-p \min \left\{c_{p}, d(p) z\right\} .\right.
$$

To derive the closed-form formula, we make a classified discussion to the following scenarios:

Case 1 If $d(q) u \leq c_{q}, d(p) u \leq c_{p}$, then (A-1) can be simplified to:

$$
\max _{l \leq z \leq u}[q d(q)-p d(p)] z .
$$

The maximum value of $(\mathrm{A}-1)$ is

$$
\left\{\begin{array}{l}
{[q d(q)-p d(p)] u, \text { if } q d(q)-p d(p) \geq 0,} \\
{[q d(q)-p d(p)] l, \text { otherwise. }}
\end{array}\right.
$$

Case 2 If $d(q) u \leq c_{q}, d(p) l \leq c_{p} \leq d(p) u$, then (A-1) can be simplified to:

$$
\max _{l \leq z \leq u} \begin{cases}{[q d(q)-p d(p)] z,} & \text { for } l \leq z<c_{q} / d(q), \\ q c_{q}-p d(p) z, & \text { for } c_{q} / d(q) \leq z \leq u .\end{cases}
$$

The maximum value of $(\mathrm{A}-1)$ is

$$
\begin{cases}q d(q) u-p c_{p}, & \text { if } q d(q)-p d(p) \geq 0, \\ \max \left\{[q d(q)-p d(p)] l, q d(q)-p c_{p}\right\}, & \text { otherwise. }\end{cases}
$$

Case 3 If $d(q) u \leq c_{q}, c_{p} \leq d(p) l$, then (A-1) can be simplified as:

$$
\max _{l \leq z \leq u} q d(q) z-p c_{p} .
$$

The maximum value of (A-1) is

$$
q d(q) u-p c_{p}
$$

Case 4 If $d(q) l \leq c_{q} \leq d(q) u, d(p) u \leq c_{p}$, then (A-1) can be simplified to:

$$
\max _{l \leq z \leq u} \begin{cases}{[q d(q)-p d(p)] z,} & \text { for } l \leq z<c_{q} / d(q), \\ q c_{q}-p d(p) z, & \text { for } c_{q} / d(q) \leq z \leq u .\end{cases}
$$

The maximum value of $(\mathrm{A}-1)$ is

$$
\begin{cases}{[q-p d(p) / d(q)] c_{q},} & \text { if } q d(q)-p d(p) \geq 0 \\ {[q d(q)-p d(p)] l,} & \text { otherwise. }\end{cases}
$$

Case 5 If $d(q) l \leq c_{q} \leq d(q) u, d(p) l \leq c_{p} \leq d(p) u$, the structure of (A-1) depends on the relative sizes of $c_{q} / d(q)$ and $c_{p} / d(p)$, thus we consider the following two subcases:

Subcase 1 Suppose $c_{q} / d(q) \leq c_{p} / d(p)$, then (A-1) can be simplified to: 


$$
\max _{l \leq z \leq u} \begin{cases}{[q d(q)-p d(p)] z,} & \text { for } l \leq z<c_{q} / d(q), \\ q c_{q}-p d(p) z, & \text { for } c_{q} / d(q) \leq z<c_{p} / d(p) . \\ q c_{q}-p c_{p}, & \text { for } c_{p} / d(p) \leq z \leq u\end{cases}
$$

The maximum value of $(\mathrm{A}-1)$ is

$$
\begin{cases}{[q-p d(p) / d(q)] c_{q},} & \text { if } q d(q)-p d(p) \geq 0 \\ {[q d(q)-p d(p)] l,} & \text { otherwise. }\end{cases}
$$

Subcase 2 Suppose $c_{q} / d(q)>c_{p} / d(p)$, then (A-1) can be simplified to:

$$
\max _{l \leq z \leq u} \begin{cases}{[q d(q)-p d(p)] z,} & \text { for } l \leq z<c_{p} / d(p), \\ q d(q) z-p c_{p}, & \text { for } c_{p} / d(p) \leq z<c_{q} / d(q), \\ q c_{q}-p c_{p}, & \text { for } c_{q} / d(q) \leq z \leq u .\end{cases}
$$

The maximum value of (A-1) is

$$
\begin{cases}q c_{q}-p c_{p}, & \text { if } q d(q)-p d(p) \geq 0 \\ \max \left\{[q d(q)-p d(p)] l, q c_{q}-p c_{p}\right\}, & \text { otherwise. }\end{cases}
$$

Case 6 If $d(q) l \leq c_{q} \leq d(q) u, c_{p} \leq d(p) l$, then (A-1) can be simplified to:

$$
\max _{l \leq z \leq u} \begin{cases}q d(q) z-p c_{p}, & \text { for } l \leq z<c_{q} / d(q), \\ q c_{q}-p c_{p}, & \text { for } c_{q} / d(q) \leq z \leq u\end{cases}
$$

The maximum value of $(\mathrm{A}-1)$ is

$$
q c_{q}-p c_{p}
$$

Case 7 If $c_{q} \leq d(q) l, d(p) u \leq c_{p}$, then (A-1) can be simplified as:

$$
\max _{l \leq z \leq u} q c_{q}-p d(p) z
$$

The maximum value of $(\mathrm{A}-1)$ is

$$
q c_{q}-p d(p) l
$$

Case 8 If $c_{q} \leq d(q) l, d(p) l \leq c_{p} \leq d(p) u$, then (A-1) can be simplified to:

$$
\max _{l \leq z \leq u} \begin{cases}q c_{q}-p d(p) z, & \text { for } l \leq z<c_{q} / d(q), \\ q c_{q}-p c_{p}, & \text { for } c_{q} / d(q) \leq z \leq u\end{cases}
$$

The maximum value of $(\mathrm{A}-1)$ is

$$
q c_{q}-p d(p) l
$$

Case 9 If $c_{q} \leq d(q) l, c_{p} \leq d(p) l$, then (A-1) can be simplified to:

$$
\max _{l \leq z \leq u} q c_{q}-p c_{p}
$$

The maximum value of (A-1) is 


$$
q c_{q}-p c_{p}
$$

In summary, the maximum value of (A-1) under any scenario is linear or piecewise linear function of $c_{p}$ and $c_{q}$.

Proof of Proposition 3 Under the conditions of Lemma 2, model (8) can be written as:

$$
\begin{array}{ll}
\max _{F} & \left\{E_{F}\left[q_{2} \min \left\{c_{q}, d\left(q_{2}\right) z\right\}-p_{2} \min \left\{c_{p}, d\left(p_{2}\right) z\right\}\right]\right. \\
\text { subject to } & \int_{l}^{u} d F=1, \\
& \int_{m}^{u} d F=\frac{1}{2}, \\
& d F \geq 0 .
\end{array}
$$

By the strong duality theorem, (A-2) can be reformulated as:

$$
\begin{array}{ll}
\min _{y_{0}, y_{1}} & \frac{1}{2}\left(y_{0}+y_{1}\right)+\frac{1}{2} y_{0} \\
\text { subject to } & y_{0}+y_{1} 1\{z \geq m\} \geq q \min \left\{d(q) z, c_{q}\right\}-p \min \left\{d(p) z, c_{p}\right\}, \quad z \in[l, u],
\end{array}
$$

where $1\{z \geq m\}$ is the indicator function, which is equal to one if $z \geq m$; otherwise, 0 .

To derive the closed-form formula, we discuss the following scenarios:

Case 1 If $d(q) u \leq c_{q}, d(p) u \leq c_{p}$, then constraint of (A-3) can be written as:

$$
y_{0}+y_{1} 1\{z \geq m\} \geq[q d(q)-p d(p)] z, \quad z \in[l, u] .
$$

Because $y_{0}+y_{1} 1\{z \geq m\}$ is a step-shaped piecewise linear function, the above equation can also be further represented as:

$$
\begin{cases}y_{0} \geq[q d(q)-p d(p)] z, & z \in[l, m), \\ y_{0}+y_{1} \geq[q d(q)-p d(p)] z, & z \in[m, u) .\end{cases}
$$

Then we obtain

$$
\min _{y_{0}, y_{1}} \frac{1}{2}\left(y_{0}+y_{1}\right)+\frac{1}{2} y_{0}=\left\{\begin{array}{l}
{[q d(q)-p d(p)](m+u) / 2, \text { if } q d(q)-p d(p) \geq 0,} \\
{[q d(q)-p d(p)](m+l) / 2, \text { if } q d(q)-p d(p)<0 .}
\end{array}\right.
$$

Case 2 If $d(q) u \leq c_{q}, d(p) l \leq c_{p} \leq d(p) u$, the constraint of (A-3) can be written as:

$$
y_{0}+y_{1} 1\{z \geq m\} \geq \begin{cases}{[q d(q)-p d(p)] z,} & \text { for } l \leq z<c_{p} / d(p), \\ q d(q) z-p c_{p}, & \text { for } c_{p} / d(p) \leq z \leq u\end{cases}
$$

Similar to the previous scenario, we consider the following two subcases:

Subcase 1 Suppose that $q d(q)-p d(p) \geq 0$,

Here, the step-shaped structure of the constraint is reliant on the relative sizes of $m$ and $c_{p} / d(p)$.

(1) When $m \geq c_{p} / d(p)$, the constraint translates to:

$$
\begin{cases}y_{0} \geq[q d(q)-p d(p)] z, & \text { for } l \leq z<c_{p} / d(p) \\ y_{0} \geq q d(q) z-p c_{p}, & \text { for } c_{p} / d(p) \leq z<m \\ y_{0}+y_{1} \geq q d(q) z-p c, & \text { for } m \leq z \leq u\end{cases}
$$


Then we obtain

$$
\min _{y_{0}, y_{1}} \frac{1}{2}\left(y_{0}+y_{1}\right)+\frac{1}{2} y_{0}=q d(q) \frac{(m+u)}{2}-p c_{p} .
$$

(2) When $m<c_{p} / d(p)$, the constraint translates to:

$$
\begin{cases}y_{0} \geq[q d(q)-p d(p)] z, & \text { for } l \leq z<m, \\ y_{0}+y_{1} \geq[q d(q)-p d(p)] z, & \text { for } m \leq z<c_{p} / d(p), \\ y_{0}+y_{1} \geq q d(q) z-p c_{p}, & \text { for } c_{p} / d(p) \leq z \leq u\end{cases}
$$

Then we obtain

$$
\min _{y_{0}, y_{1}} \frac{1}{2}\left(y_{0}+y_{1}\right)+\frac{1}{2} y_{0}=[q d(q)-p d(p)] \frac{m}{2}+\frac{q d(q) u-p c_{p}}{2} .
$$

Subcase 2 Suppose $q d(q)-p d(p)<0$,

(1) When $m \geq c_{p} / d(p)$, the constraint translates to:

$$
\left\{\begin{array}{l}
y_{0} \geq[q d(q)-p d(p)] z, \text { for } l \leq z \leq c_{p} / d(p) \\
y_{0} \geq q d(q) z-p c_{p}, \quad \text { for } c_{p} / d(p) \leq z \leq m \\
y_{0}+y_{1} \geq q d(q) z-p c, \text { for } m \leq z \leq u
\end{array}\right.
$$

Then we obtain

$$
\min _{y_{0}, y_{1}} \frac{1}{2}\left(y_{0}+y_{1}\right)+\frac{1}{2} y_{0}=\max \left\{\frac{[q d(q)-p d(p)] l}{2}+\frac{q d(q) u-p c_{p}}{2}, q d(q) \frac{m+u}{2}-p c_{p}\right\} .
$$

(2) When $m<c_{p} / d(p)$, the constraint translates to:

$$
\begin{cases}y_{0} \geq[q d(q)-p d(p)] z, & \text { for } l \leq z<m, \\ y_{0}+y_{1} \geq[q d(q)-p d(p)] z, & \text { for } m \leq z<c_{p} / d(p) \\ y_{0}+y_{1} \geq q d(q) z-p c_{p}, & \text { for } c_{p} / d(p) \leq z \leq u\end{cases}
$$

Then we obtain

$$
\min _{y_{0}, y_{1}} \frac{1}{2}\left(y_{0}+y_{1}\right)+\frac{1}{2} y_{0}=\max \left\{\frac{[q d(q)-p d(p)] l}{2}+\frac{q d(q) u-p c_{p}}{2},[q d(q)-p d(p)] \frac{m+l}{2}\right\} \text {. }
$$

Case 3 If $d(q) u \leq c_{q}, c_{p} \leq d(p) l$, constraint of (A-3) can be written as:

$$
y_{0}+y_{1} 1\{z \geq m\} \geq q d(q) z-p c_{p}, \quad \forall z \in[l, u] .
$$

Since $y_{0}+y_{1} 1\{z \geq m\}$ is a step-shaped piecewise linear function, the above equation can be represented further as:

$$
\begin{cases}y_{0} \geq q d(q) z-p c_{p}, & \text { for } z \in[l, m) \\ y_{0}+y_{1} \geq q d(q) z-p c_{p}, & \text { for } z \in[m, u] .\end{cases}
$$


Then we obtain

$$
\min _{y_{0}, y_{1}} \frac{1}{2}\left(y_{0}+y_{1}\right)+\frac{1}{2} y_{0}=q d(q) \frac{m+u}{2}-p c_{p} .
$$

Case 4 If $d(q) l \leq c_{q} \leq d(q) u, d(p) u \leq c_{p}$, constraint of (A-3) can be written as:

$$
y_{0}+y_{1} 1\{z \geq m\} \geq \begin{cases}{[q d(q)-p d(p)] z,} & \text { for } l \leq z \leq c_{q} / d(q), \\ q c_{q}-p d(p) z, & \text { for } c_{q} / d(q) \leq z \leq u .\end{cases}
$$

Consider the following two subcases:

Subcase 1 Suppose that $q d(q)-p d(p) \geq 0$,

Under the subcase, the step-shaped structure of the constraint further relies on the relative sizes of $m$ and $c_{q} / d(q)$.

(1) When $m \geq c_{q} / d(q)$, the constraint translates to:

$$
\begin{cases}y_{0} \geq[q d(q)-p d(p)] z, & \text { for } l \leq z \leq c_{q} / d(q), \\ y_{0} \geq q c_{q}-p d(p) z, & \text { for } c_{q} / d(q) \leq z \leq m, \\ y_{0}+y_{1} \geq q c_{q}-p d(p) z, & \text { for } m \leq z \leq u\end{cases}
$$

Then we obtain

$$
\min _{y_{0}, y_{1}} \frac{1}{2}\left(y_{0}+y_{1}\right)+\frac{1}{2} y_{0}=q c_{q}-p d(p) \frac{\left(m+c_{q} / d(q)\right)}{2} .
$$

(2) When $m<c_{q} / d(q)$, the constraint translates to:

$$
\begin{cases}y_{0} \geq[q d(q)-p d(p)] z, & \text { for } l \leq z \leq m, \\ y_{0}+y_{1} \geq[q d(q)-p d(p)] z, & \text { for } m \leq z \leq c_{q} / d(q), \\ y_{0}+y_{1} \geq q c_{q}-p d(p) z, & \text { for } c_{q} / d(q) \leq z \leq u .\end{cases}
$$

Then we obtain

$$
\min _{y_{0}, y_{1}} \frac{1}{2}\left(y_{0}+y_{1}\right)+\frac{1}{2} y_{0}=[q d(q)-p d(p)] \frac{m+c_{q} / d(q)}{2} .
$$

Subcase 2 Suppose that $q d(q)-p d(p)<0$,

(1) When $m \geq c_{q} / d(q)$, the constraint translates to:

$$
\begin{cases}y_{0} \geq[q d(q)-p d(p)] z, & \text { for } l \leq z \leq c_{q} / d(q), \\ y_{0} \geq q c_{q}-p d(p) z, & \text { for } c_{q} / d(q) \leq z \leq m, \\ y_{0}+y_{1} \geq q c_{q}-p d(p) z, & \text { for } m \leq z \leq u\end{cases}
$$

Then we obtain

$$
\min _{y_{0}, y_{1}} \frac{1}{2}\left(y_{0}+y_{1}\right)+\frac{1}{2} y_{0}=q \frac{c_{q}+d(q) l}{2}-p d(p) \frac{m+l}{2} .
$$


(2) When $m<c_{q} / d(q)$, the constraint translates to:

$$
\begin{cases}y_{0} \geq[q d(q)-p d(p)] z, & \text { for } l \leq z \leq m, \\ y_{0}+y_{1} \geq[q d(q)-p d(p)] z, & \text { for } m \leq z<c_{q} / d(q), \\ y_{0}+y_{1} \geq q c_{q}-p d(p) z, & \text { for } c_{q} / d(q) \leq z \leq u\end{cases}
$$

Then we obtain

$$
\min _{y_{0}, y_{1}} \frac{1}{2}\left(y_{0}+y_{1}\right)+\frac{1}{2} y_{0}=[q d(q)-p d(p)] \frac{m+l}{2} .
$$

Case 5 If $d(q) l \leq c_{q} \leq d(q) u, d(p) l \leq c_{p} \leq d(p) u$, to recognize the structure of the constraint, again, we consider the two subcases, namely $q d(q)-p d(p) \geq 0$ and $q d(q)-p d(p)<0$. The challenge lies in further consideration of full permutation of three values $m, c_{q} / d(q)$ and $c_{p} / d(p)$ under each subcase. Accordingly, we have $2 \times A_{3}^{3}=12$ scenarios.

Subcase 1 Suppose that $q d(q)-p d(p) \geq 0$,

(1) When $c_{q} / d(q) \leq m<c_{p} / d(p)$, the constraint translates to:

$$
\begin{cases}y_{0} \geq[q d(q)-p d(p)] z, & \text { for } l \leq z<c_{q} / d(q), \\ y_{0} \geq q c_{q}-p d(p) z, & \text { for } c_{q} / d(q) \leq z<m, \\ y_{0}+y_{1} \geq q c_{q}-p d(p) z, & \text { for } m \leq z<c_{p} / d(p), \\ y_{0}+y_{1} \geq q c_{q}-p c_{p}, & \text { for } c_{p} / d(p) \leq z \leq u\end{cases}
$$

Then we obtain

$$
\min _{y_{0}, y_{1}} \frac{1}{2}\left(y_{0}+y_{1}\right)+\frac{1}{2} y_{0}=q c_{q}-p d(p) \frac{c_{q} / d(q)+m}{2} .
$$

(2) When $c_{q} / d(q) \leq c_{p} / d(p)<m$, the constraint tranlates to:

$$
\begin{cases}y_{0} \geq[q d(q)-p d(p)] z, & \text { for } l \leq z<c_{q} / d(q), \\ y_{0} \geq q c_{q}-p d(p) z, & \text { for } c_{q} / d(q) \leq z<c_{p} / d(p), \\ y_{0} \geq q c_{q}-p c_{p}, & \text { for } c_{p} / d(p) \leq z<m, \\ y_{0}+y_{1} \geq q c_{q}-p c_{p}, & \text { for } m \leq z<u\end{cases}
$$

Then we obtain

$$
\min _{y_{0}, y_{1}} \frac{1}{2}\left(y_{0}+y_{1}\right)+\frac{1}{2} y_{0}=[q d(q)-p d(p)] \frac{c_{q}}{2 d(q)}+\frac{q c_{q}-p c_{p}}{2} .
$$

(3) When $c_{p} / d(p) \leq m<c_{q} / d(q)$, the constraint tranlates to:

$$
\begin{cases}y_{0} \geq[q d(q)-p d(p)] z, & \text { for } l \leq z<c_{p} / d(p), \\ y_{0} \geq q d(q) z-p c_{p}, & \text { for } c_{p} / d(p) \leq z<m \\ y_{0} \geq q d(q) z-p c_{p}, & \text { for } m \leq z<c_{q} / d(q) \\ y_{0}+y_{1} \geq q c_{q}-p c_{p}, & \text { for } c_{q} / d(q) \leq z<u\end{cases}
$$

Then we obtain 


$$
\min _{y_{0}, y_{1}} \frac{1}{2}\left(y_{0}+y_{1}\right)+\frac{1}{2} y_{0}=q \frac{c_{q}+d(q) m}{2}-p c_{p} .
$$

(4) When $c_{p} / d(p) \leq c_{q} / d(q)<m$, the constraint tranlates to:

$$
\begin{cases}y_{0} \geq[q d(q)-p d(p)] z, & \text { for } l \leq z<c_{p} / d(p), \\ y_{0} \geq q d(q) z-p c_{p}, & \text { for } c_{p} / d(p) \leq z<c_{q} / d(q), \\ y_{0} \geq q c_{q}-p c_{p}, & \text { for } c_{q} / d(q) \leq z<m, \\ y_{0}+y_{1} \geq q c_{q}-p c_{p}, & \text { for } m \leq z \leq u .\end{cases}
$$

Then we obtain

$$
\min _{y_{0}, y_{1}} \frac{1}{2}\left(y_{0}+y_{1}\right)+\frac{1}{2} y_{0}=q c_{q}-p c_{p} .
$$

(5) When $m \leq c_{q} / d(q)<c_{p} / d(p)$, the constraint tranlates to:

$$
\begin{cases}y_{0} \geq[q d(q)-p d(p)] z, & \text { for } l \leq z<m, \\ y_{0}+y_{1} \geq[q d(q)-p d(p)] z, & \text { for } m \leq z<c_{q} / d(q), \\ y_{0}+y_{1} \geq q c_{q}-p d(p) z, & \text { for } c_{q} / d(q) \leq z<c_{p} / d(p), \\ y_{0}+y_{1} \geq q c_{q}-p c_{p}, & \text { for } c_{p} / d(p) \leq z \leq u\end{cases}
$$

Then we obtain

$$
\min _{y_{0}, y_{1}} \frac{1}{2}\left(y_{0}+y_{1}\right)+\frac{1}{2} y_{0}=[q d(q)-p d(p)] \frac{m+c_{q} / d(q)}{2} .
$$

(6) When $m \leq c_{p} / d(p)<c_{q} / d(q)$, the constraint tranlates to:

$$
\begin{cases}y_{0} \geq[q d(q)-p d(p)] z, & \text { for } l \leq z<m, \\ y_{0}+y_{1} \geq[q d(q)-p d(p)] z, & \text { for } m \leq z<c_{p} / d(p), \\ y_{0}+y_{1} \geq q d(q) z-p c_{p}, & \text { for } c_{p} / d(p) \leq z<c_{q} / d(q), \\ y_{0}+y_{1} \geq q c_{q}-p c_{p}, & \text { for } c_{q} / d(q) \leq z \leq u .\end{cases}
$$

Then we obtain

$$
\min _{y_{0}, y_{1}} \frac{1}{2}\left(y_{0}+y_{1}\right)+\frac{1}{2} y_{0}=[q d(q)-p d(p)] \frac{m+c_{q} / d(q)}{2} .
$$

Subcase 2 Suppose that $q d(q)-p d(p)<0$,

(1) When $c_{q} / d(q) \leq m<c_{p} / d(p)$, the constraint translates to:

$$
\begin{cases}y_{0} \geq[q d(q)-p d(p)] z, & \text { for } l \leq z<c_{q} / d(q), \\ y_{0} \geq q c_{q}-p d(p) z, & \text { for } c_{q} / d(q) \leq z<m, \\ y_{0}+y_{1} \geq q c_{q}-p d(p) z, & \text { for } m \leq z<c_{p} / d(p), \\ y_{0}+y_{1} \geq q c_{q}-p c_{p}, & \text { for } c_{p} / d(p) \leq z \leq u\end{cases}
$$

Then we obtain

$$
\min _{y_{0}, y_{1}} \frac{1}{2}\left(y_{0}+y_{1}\right)+\frac{1}{2} y_{0}=[q d(q)-p d(p)] \frac{l}{2}+\frac{q c_{q}-p d(p) m}{2} .
$$


(2) When $c_{q} / d(q) \leq c_{p} / d(p)<m$, the constraint tranlates to:

$$
\begin{cases}y_{0} \geq[q d(q)-p d(p)] z, & \text { for } l \leq z<c_{q} / d(q), \\ y_{0} \geq q c_{q}-p d(p) z, & \text { for } c_{q} / d(q) \leq z<c_{p} / d(p), \\ y_{0} \geq q c_{q}-p c_{p}, & \text { for } c_{p} / d(p) \leq z<m, \\ y_{0}+y_{1} \geq q c_{q}-p c_{p}, & \text { for } m \leq z \leq u .\end{cases}
$$

Then we obtain

$$
\min _{y_{0}, y_{1}} \frac{1}{2}\left(y_{0}+y_{1}\right)+\frac{1}{2} y_{0}=[q d(q)-p d(p)] \frac{l}{2}+\frac{q c_{q}-p c_{p}}{2} .
$$

(3) When $c_{p} / d(p) \leq m<c_{q} / d(q)$, the constraint tranlates to:

$$
\begin{cases}y_{0} \geq[q d(q)-p d(p)] z, & \text { for } l \leq z<c_{p} / d(p) \\ y_{0} \geq q d(q) z-p c_{p}, & \text { for } c_{p} / d(p) \leq z<m \\ y_{0} \geq q d(q) z-p c_{p}, & \text { for } m \leq z<c_{q} / d(q) \\ y_{0}+y_{1} \geq q c_{q}-p c_{p}, & \text { for } c_{q} / d(q) \leq z \leq u\end{cases}
$$

Then we obtain

$$
\min _{y_{0}, y_{1}} \frac{1}{2}\left(y_{0}+y_{1}\right)+\frac{1}{2} y_{0}=\max \left\{q \frac{c_{q}+d(q) m}{2}-p c_{p},[q d(q)-p d(p)] \frac{l}{2}+\frac{q c_{q}-p c_{p}}{2}\right\} \text {. }
$$

(4) When $c_{p} / d(p) \leq c_{q} / d(q)<m$, the constraint tranlates to:

$$
\begin{cases}y_{0} \geq[q d(q)-p d(p)] z, & \text { for } l \leq z<c_{p} / d(p), \\ y_{0} \geq q d(q) z-p c_{p}, & \text { for } c_{p} / d(p) \leq z<c_{q} / d(q), \\ y_{0} \geq q c_{q}-p c_{p}, & \text { for } c_{q} / d(q) \leq z<m, \\ y_{0}+y_{1} \geq q c_{q}-p c_{p}, & \text { for } m \leq z \leq u\end{cases}
$$

Then we obtain

$$
\min _{y_{0}, y_{1}} \frac{1}{2}\left(y_{0}+y_{1}\right)+\frac{1}{2} y_{0}=\max \left\{q c_{q}-p c_{p},[q d(q)-p d(p)] \frac{l}{2}+\frac{q c_{q}-p c_{p}}{2}\right\} .
$$

(5) When $m \leq c_{q} / d(q)<c_{p} / d(p)$, the constraint tranlates to:

$$
\begin{cases}y_{0} \geq[q d(q)-p d(p)] z, & \text { for } l \leq z<m, \\ y_{0}+y_{1} \geq[q d(q)-p d(p)] z, & \text { for } m \leq z<c_{q} / d(q), \\ y_{0}+y_{1} \geq q c_{q}-p d(p) z, & \text { for } c_{q} / d(q) \leq z<c_{p} / d(p), \\ y_{0}+y_{1} \geq q c_{q}-p c_{p}, & \text { for } c_{p} / d(p) \leq z \leq u\end{cases}
$$

Then we obtain

$$
\min _{y_{0}, y_{1}} \frac{1}{2}\left(y_{0}+y_{1}\right)+\frac{1}{2} y_{0}=[q d(q)-p d(p)] \frac{m+l}{2} .
$$

(6) When $m \leq c_{p} / d(p)<c_{q} / d(q)$, the constraint tranlates to: 


$$
\begin{cases}y_{0} \geq[q d(q)-p d(p)] z, & \text { for } l \leq z<m \\ y_{0}+y_{1} \geq[q d(q)-p d(p)] z, & \text { for } m \leq z<c_{p} / d(p) \\ y_{0}+y_{1} \geq q d(q) z-p c_{p}, & \text { for } c_{p} / d(p) \leq z<c_{q} / d(q) \\ y_{0}+y_{1} \geq q c_{q}-p c_{p}, & \text { for } c_{q} / d(q) \leq z \leq u\end{cases}
$$

Then we obtain

$$
\min _{y_{0}, y_{1}} \frac{1}{2}\left(y_{0}+y_{1}\right)+\frac{1}{2} y_{0}=\max \left\{[q d(q)-p d(p)] \frac{m+l}{2},[q d(q)-p d(p)] \frac{l}{2}+\frac{q c_{q}-p c_{p}}{2}\right\} .
$$

Case 6 If $d(q) l \leq c_{q} \leq d(q) u, c_{p} \leq d(p) l$, constraint of (A-3) can be written as:

$$
y_{0}+y_{1} 1\{z \geq m\} \geq \begin{cases}q d(q) z-p c_{p}, & \text { for } l \leq z<c_{q} / d(q), \\ q c_{q}-p c_{p}, & \text { for } c_{q} / d(q) \leq z \leq u .\end{cases}
$$

Next consider the relative sizes of $m$ and $c_{q} / d(q)$ :

Subcase 1 If $m \geq c_{q} / d(q)$, the constraint translates to:

$$
\begin{cases}y_{0} \geq q d(q) z-p c_{p}, & \text { for } l \leq z<c_{q} / d(q) \\ y_{0} \geq q c_{q}-p c_{p}, & \text { for } c_{q} / d(q) \leq z<m \\ y_{0}+y_{1} \geq q c_{q}-p c_{p}, & \text { for } m \leq z \leq u\end{cases}
$$

Then we obtain

$$
\min _{y_{0}, y_{1}} \frac{1}{2}\left(y_{0}+y_{1}\right)+\frac{1}{2} y_{0}=q c_{q}-p c_{p} .
$$

Subcase 2 If $m<c_{q} / d(q)$, the constraint translates to:

$$
\begin{cases}y_{0} \geq q d(q) z-p c_{p}, & \text { for } l \leq z<m \\ y_{0}+y_{1} \geq q d(q) z-p c_{p}, & \text { for } m \leq z<c_{q} / d(q), \\ y_{0}+y_{1} \geq q c_{q}-p c_{p}, & \text { for } c_{q} / d(q) \leq z \leq u\end{cases}
$$

Then we obtain

$$
\min _{y_{0}, y_{1}} \frac{1}{2}\left(y_{0}+y_{1}\right)+\frac{1}{2} y_{0}=q \frac{c_{q}+d(q) m}{2}-p c_{p} .
$$

Case 7 If $c_{q} \leq d(q) l, d(p) u \leq c_{p}$, constraint of (A-3) can be written as:

$$
y_{0}+y_{1} 1\{z \geq m\} \geq q c_{q}-p d(p) z .
$$

Then we obtain

$$
\min _{y_{0}, y_{1}} \frac{1}{2}\left(y_{0}+y_{1}\right)+\frac{1}{2} y_{0}=q c_{q}-p d(p) \frac{m+l}{2} .
$$

Case 8 If $c_{q} \leq d(q) l, d(p) l \leq c_{p} \leq d(p) u$, constraint of (A-3) can be written as: 


$$
y_{0}+y_{1} 1\{z \geq m\} \geq \begin{cases}q c_{q}-p d(p) z, & \text { for } l \leq z<c_{p} / d(p), \\ q c_{q}-p c_{p}, & \text { for } c_{p} / d(p) \leq z \leq u .\end{cases}
$$

Next consider the relative sizes of $m$ and $c_{p} / d(p)$ :

Subcase 1 If $m \geq c_{p} / d(p)$, the constraint translates to:

$$
\begin{cases}y_{0} \geq q c_{q}-p d(p) z, & \text { for } l \leq z<c_{p} / d(p), \\ y_{0} \geq q c_{q}-p c_{p}, & \text { for } c_{p} / d(p) \leq z<m, \\ y_{0}+y_{1} \geq q c_{q}-p c_{p}, & \text { for } m \leq z<u\end{cases}
$$

Then we obtain

$$
\min _{y_{0}, y_{1}} \frac{1}{2}\left(y_{0}+y_{1}\right)+\frac{1}{2} y_{0}=q c_{q}-p \frac{d(p) l+c_{p}}{2} .
$$

Subcase 2 If $m<c_{p} / d(p)$, the constraint translates to:

$$
\begin{cases}y_{0} \geq q c_{q}-p d(p) z, & \text { for } l \leq z<m \\ y_{0}+y_{1} \geq q c_{q}-p d(p) z, & \text { for } m \leq z<c_{p} / d(p) \\ y_{0}+y_{1} \geq q c_{q}-p c_{p}, & \text { for } c_{p} / d(p) \leq z<u\end{cases}
$$

Then we obtain

$$
\min _{y_{0}, y_{1}} \frac{1}{2}\left(y_{0}+y_{1}\right)+\frac{1}{2} y_{0}=q c_{q}-p d(p) \frac{m+l}{2} .
$$

Case 9 If $c_{q} \leq d(q) l, c_{p} \leq d(p) l$, constraint of (A-3) can be written as:

$$
y_{0}+y_{1} 1\{z \geq m\} \geq q c_{q}-p c_{p} .
$$

Then

$$
\min _{y_{0}, y_{1}} \frac{1}{2}\left(y_{0}+y_{1}\right)+\frac{1}{2} y_{0}=q c_{q}-p c_{p} .
$$

Based on the above observation, we can deduce that the closed-form solutions to the problem (9) in each small domain $\Delta t$ are either linear functions or piecewise linear functions of $c_{p}$ and $c_{q}$.

Proof of Proposition 4 By the strong duality theorem, the dual form of model (9) is represented as follows:

$$
\begin{aligned}
& \min _{y_{0}, y_{1}} \quad y_{0}+y_{1} \\
& \text { subject to } 2 y_{0}+y_{1} \geq q \min \left\{d(q)(m-z), c_{q}\right\} \\
& \quad-p \min \left\{d(p)(m-z), c_{q}\right\} \\
& \quad+q \min \left\{d(q)(m+z), c_{q}\right\} \\
& \quad-p \min \left\{d(p)(m+z), c_{q}\right\}, \quad 0 \leq z \leq m .
\end{aligned}
$$

The constraint of model (A-4) is equivalent to the linear programming as follows: 


$$
\begin{aligned}
\frac{1}{2} \max _{0 \leq z \leq m}\{ & q \min \left\{d(q)(m-z), c_{q}\right\} \\
& -p \min \left\{d(p)(m-z), c_{q}\right\} \\
& +q \min \left\{d(q)(m+z), c_{q}\right\} \\
& -p \min \left\{d(p)(m+z), c_{p}\right\} .
\end{aligned}
$$

Let

$$
\begin{aligned}
& \varphi\left(z \mid q, c_{q}\right)=\min \left\{d(q)(m-z), c_{q}\right\}+\min \left\{d(q)(m+z), c_{q}\right\} . \\
& \varphi\left(z \mid p, c_{p}\right)=\min \left\{d(p)(m-z), c_{p}\right\}+\min \left\{d(p)(m+z), c_{p}\right\} .
\end{aligned}
$$

Then (A-5) is represented as follows:

$$
\frac{1}{2} \max _{0 \leq z \leq m} q \varphi\left(z \mid q, c_{q}\right)-p \varphi\left(z \mid p, c_{p}\right) .
$$

Function $\varphi\left(z \mid p, c_{p}\right)$ exhibits different formations depending on the relative sizes of $c_{p} / d(p), m$, and $2 m$.

Case $1 c_{p} / d(p) \geq m, c_{q} / d(q) \geq m$.

The shape of function $q \varphi\left(z \mid q, c_{q}\right)-p \varphi\left(z \mid p, c_{p}\right)$ further relies on the relative sizes of $2 d(p) m$ and $2 d(q) m$. Thus, we consider the following subcases:

(1) If $c_{p} \leq 2 d(p) m, c_{q} \leq 2 d(q) m$,

$\frac{1}{2} \max _{0 \leq z \leq m}\left\{q \varphi\left(z \mid q, c_{q}\right)\right.$,

$= \begin{cases}\max \left\{[q d(q)-p d(p)] m,[q d(q)-p d(p)] m+\frac{p d(p) c_{q}}{2 d(q)}-\frac{p c_{p}}{2}\right\}, & \text { if } q d(q)-p d(p) \geq 0, \\ {[q d(q)-p d(p)] m} & \text { if } q d(q)-p d(p)<0, \frac{c_{p}}{d(p)} \geq \frac{c_{q}}{d(q)}, q c_{q}-p c_{p} \leq 2[q d(q)-p d(p)] m, \\ \frac{q c_{q}-p c_{p}}{2}, & \text { otherwise. }\end{cases}$

(2) If $c_{p} \leq 2 d(p) m, c_{q}>2 d(q) m$,

$$
\frac{1}{2} \max _{0 \leq z \leq m}\left\{q \varphi\left(z \mid q, c_{q}\right)-p \varphi\left(z \mid p, c_{p}\right)\right\}=q d(q) m-p c_{p} / 2 .
$$

(3) If $c_{p}>2 d(p) m, c_{q} \leq 2 d(q) m$,

$$
\frac{1}{2} \max _{0 \leq z \leq m}\left\{q \varphi\left(z \mid q, c_{q}\right)-p \varphi\left(z \mid p, c_{p}\right)\right\}=[q d(q)-p d(p)] m .
$$

(4) If $c_{p}>2 d(p) m, c_{q}>2 d(q) m$,

$$
\frac{1}{2} \max _{0 \leq z \leq m}\left\{q \varphi\left(z \mid q, c_{q}\right)-p \varphi\left(z \mid p, c_{p}\right)\right\}=[q d(q)-p d(p)] m .
$$

Case $2 c_{p} / d(p)<m, c_{q} / d(q) \geq m$,

(1) If $c_{q} \leq 2 d(q) m$ 
$\frac{1}{2} \max _{0 \leq z \leq m}\left\{q \varphi\left(z \mid q, c_{q}\right)-p \varphi\left(z \mid p, c_{p}\right)\right\}$

$$
= \begin{cases}\max \left\{[q d(q)-p d(p)] m+\frac{p d(p) c_{q}}{2 d(q)}-\frac{p c_{p}}{2}, q d(q) m-p c_{p}\right\}, & \text { if } q d(q)-p d(p) \geq 0, \\ q d(q) m-p c_{p}, & \text { if } q d(q)-p d(p)<0, \\ \frac{q c_{q}-p c_{p}}{2}, & \frac{c_{p}}{d(p)}+\frac{c_{q}}{d(q)} \leq 2 m, \\ & q c_{q}+p c_{p} \leq 2 q d(q) m, \\ & \text { otherwise. }\end{cases}
$$

(2) If $c_{q}>2 d(q) m$

$$
\frac{1}{2} \max _{0 \leq z \leq m}\left\{q \varphi\left(z \mid q, c_{q}\right)-p \varphi\left(z \mid p, c_{p}\right)\right\}=q d(q) m-p c_{p} .
$$

Case $3 c_{p} / d(p) \geq m, c_{q} / d(q)<m$,

(1) If $c_{q} \leq 2 d(p) m$

$\frac{1}{2} \max _{0 \leq z \leq m}\left\{q \varphi\left(z \mid q, c_{q}\right)-p \varphi\left(z \mid p, c_{p}\right)\right\}$

$$
= \begin{cases}\max \left\{\left[q c_{q}-\frac{p d(p) c_{q}}{2 d(q)}-\frac{p c_{p}}{2}, q c_{q}-p d(p) m\right\},\right. & \text { if } q d(q)-p d(p) \geq 0, \\ q c_{q}-p d(p) m, & \text { if } q d(q)-p d(p)<0, \\ \frac{q c_{q}-p c_{p}}{2}, & \frac{c_{p}}{d(p)}+\frac{c_{q}}{d(q)} \geq 2 m, \\ & q c_{q}+p c_{p} \leq 2 p d(p) m, \\ & \text { otherwise. }\end{cases}
$$

(2) If $c_{p}>2 d(p) m$

$$
\frac{1}{2} \max _{0 \leq z \leq m}\left\{q \varphi\left(z \mid q, c_{q}\right)-p \varphi\left(z \mid p, c_{p}\right)\right\}=q c_{q}-p d(p) m .
$$

Case $4 c_{p} / d(p)<m, c_{q} / d(q)<m$,

(1) If $q d(q) \geq p d(p)$

$$
\frac{1}{2} \max _{0 \leq z \leq m}\left\{q \varphi\left(z \mid q, c_{q}\right)-p \varphi\left(z \mid p, c_{p}\right)\right\}=\max \left\{\left[q c_{q}-p c_{p}, q c_{q}-\frac{p d(p) c_{q}}{2 d(q)}-\frac{p c_{p}}{2}\right\} .\right.
$$

(2) If $q d(q)<p d(p)$

$$
\frac{1}{2} \max _{0 \leq z \leq m}\left\{q \varphi\left(z \mid q, c_{q}\right)-p \varphi\left(z \mid p, c_{p}\right)\right\}= \begin{cases}q c_{q}-p c_{p}, & \text { if } q c_{q} \leq p c_{p}, \frac{c_{q}}{d(q)} \geq \frac{c_{p}}{d(p)}, \\ \frac{q c_{q}-p c_{p}}{2}, & \text { otherwise. }\end{cases}
$$

In conclusion, the closed-form formulae for problem (9) in each small domain $\Delta t$ are either linear functions or piecewise linear functions of $c_{p}$ and $c_{q}$. 
Acknowledgements The authors would like to acknowledge the support from the Research center for Smarter Supply chain and Jiangsu Center for Research in Contemporary Finance at Soochow University. Funding was provided by the National Social Science Funds for Major Projects (Grant No. 18ZDA059).

\section{References}

Aissi, H., Bazgan, C., \& Vanderpooten, D. (2007). Approximation of min-max and min-max regret versions of some combinatorial optimization problems. European Journal of Operational Research, $179(2), 281-290$.

Aissi, H., Bazgan, C., \& Vanderpooten, D. (2009). Min-max and min-max regret versions of combinatorial optimization problems: A survey. European Journal of Operational Research, 197(2), 427-438.

Alaiz-RodrÃguez, R., Guerrero-Curieses, A., \& Cid-Sueiro, J. (2007). Minimax regret classifier for imprecise class distributions. Journal of Machine Learning Research, 8(Jan), 103-130.

Amaruchkul, K., Cooper, W. L., \& Gupta, D. (2007). Single-leg air-cargo revenue management. Transportation Science, 41(4), 457-469.

Amaruchkul, K., Cooper, W. L., \& Gupta, D. (2011). A note on air-cargo capacity contracts. Production and Operations Management, 20(1), 152-162.

Amaruchkul, K., \& Lorchirachoonkul, V. (2011). Air-cargo capacity allocation for multiple freight forwarders. Transportation Research Part E: Logistics and Transportation Review, 47(1), 30-40.

Bartodziej, P., Derigs, U., Malcherek, D., \& Vogel, U. (2009). Models and algorithms for solving combined vehicle and crew scheduling problems with rest constraints: An application to road feeder service planning in air cargo transportation. OR Spectrum, 31(2), 405-429.

Bartók, G., Foster, D. P., Pál, D., Rakhlin, A., \& Szepesvári, C. (2014). Partial monitoring-Classification, regret bounds, and algorithms. Mathematics of Operations Research, 39(4), 967-997.

Bertsimas, D., Brown, D. B., \& Caramanis, C. (2011). Theory and applications of robust optimization. SIAM Review, 53(3), 464-501.

Bertsimas, D., \& Popescu, I. (2005). Optimal inequalities in probability theory: A convex optimization approach. SIAM Journal on Optimization, 15(3), 780-804.

Besbes, O., \& Zeevi, A. (2009). Dynamic pricing without knowing the demand function: Risk bounds and near-optimal algorithms. Operations Research, 57(6), 1407-1420.

Boeing Company. (2018). World Air Cargo forecast 2018-2037. Retrieved March 22, 2019 http://www. boeing.com.

Caçador, S. C., Godinho, P. M. C., \& Dias, J. M. P. C. M. (2020). A minimax regret portfolio model based on the investor's utility loss. Operational Research, 1-36. https://doi.org/10.1007/s1235 1-020-00550-0.

Campos, J. R., Assunção, E., Silva, G. N., Lodwick, W. A., \& Teixeira, M. C. (2019). Discrete-time interval optimal control problem. International Journal of Control, 92(8), 1778-1784.

Chen, B., Wang, J., Wang, L., He, Y., \& Wang, Z. (2014). Robust optimization for transmission expansion planning: Minimax cost versus minimax regret. IEEE Transactions on Power Systems, 29(6), 3069-3077.

Chen, Y., \& Farias, V. F. (2018). Robust dynamic pricing with strategic customers. Mathematics of Operations Research, 43(4), 1119-1142.

Chu, J., Huang, K., \& Thiele, A. (2019). A robust optimization approach to model supply and demand uncertainties in inventory systems. Journal of the Operational Research Society, 70(11), 1885-1899.

Dong, C., Huang, G. H., Cai, Y. P., \& Xu, Y. (2011). An interval-parameter minimax regret programming approach for power management systems planning under uncertainty. Applied Energy, 88(8), 2835-2845.

Eldar, Y. C., \& Merhav, N. (2004). A competitive minimax approach to robust estimation of random parameters. IEEE Transactions on Signal Processing, 52(7), 1931-1946.

Feng, B., Li, Y., \& Shen, Z. J. M. (2015). Air cargo operations: Literature review and comparison with practices. Transportation Research Part C: Emerging Technologies, 56, 263-280.

Giove, S., Funari, S., \& Nardelli, C. (2006). An interval portfolio selection problem based on regret function. European Journal of Operational Research, 170(1), 253-264.

Gupta, D. (2008). Flexible carrier-forwarder contracts for air cargo business. Journal of Revenue and Pricing Management, 7(4), 341-356. 
Han, D. L., Tang, L. C., \& Huang, H. C. (2010). A Markov model for single-leg air cargo revenue management under a bid-price policy. European Journal of Operational Research, 200(3), 800-811.

Hayashi, T. (2008). Regret aversion and opportunity dependence. Journal of Economic Theory, 139(1), $242-268$.

Hellermann, R., Huchzermeier, A., \& Spinler, S. (2013). Options contracts with overbooking in the air cargo industry. Decision Sciences, 44(2), 297-327.

Jiang, R., Wang, J., Zhang, M., \& Guan, Y. (2013). Two-stage minimax regret robust unit commitment. IEEE Transactions on Power Systems, 28(3), 2271-2282.

Jo, H. C., \& Joo, S. K. (2015). Superconducting fault current limiter placement for power system protection using the minimax regret criterion. IEEE Transactions on Applied Superconductivity, 25(3), $1-5$.

Kasilingam, R. G. (1997a). Air cargo revenue management: Characteristics and complexities. European Journal of Operational Research, 96(1), 36-44.

Kasilingam, R. G. (1997b). An economic model for air cargo overbooking under stochastic capacity. Computers \& Industrial Engineering, 32(1), 221-226.

Kazakçi, A. O., Rozakis, S., \& Vanderpooten, D. (2007). Energy crop supply in France: A min-max regret approach. Journal of the Operational Research Society, 58(11), 1470-1479.

Lange, A. (2019). Does cargo matter? The impact of air cargo operations on departure on-time performance for combination carriers. Transportation Research Part A: Policy and Practice, 119, 214-223.

Leung, L. C., Van Hui, Y., Wang, Y., \& Chen, G. (2009). A 0-1 LP model for the integration and consolidation of air cargo shipments. Operations Research, 57(2), 402-412.

Levin, Y., Nediak, M., \& Topaloglu, H. (2012). Cargo capacity management with allotments and spot market demand. Operations Research, 60(2), 351-365.

Li, J., \& Wang, L. (2020). A minimax regret approach for robust multi-objective portfolio selection problems with ellipsoidal uncertainty sets. Computers \& Industrial Engineering, 147, 106646

Li, Y., Li, Z., Wen, F., \& Shahidehpour, M. (2018). Minimax-regret robust co-optimization for enhancing the resilience of integrated power distribution and natural gas systems. IEEE Transactions on Sustainable Energy, 11(1), 61-71.

Lim, A., Shanthikumar, J. G., \& Watewai, T. (2008). Robust multi-product pricing. Retrieved at SSRN. https://ssrn.com/abstract=1078012 or https://doi.org/10.2139/ssrn.1078012.

Lim, A. E., \& Shanthikumar, J. G. (2007). Relative entropy, exponential utility, and robust dynamic pricing. Operations Research, 55(2), 198-214.

Malladi, S. S., Erera, A. L., \& White III, C. C. (2018). Inventory control with modulated demand and a partially observed modulation process. arXiv preprint arXiv:1803.06742.

Milnor, J. (1951). Games against nature (No. RAND-RM-679). RAND PROJECT AIR FORCE SANTA MONICA CA.

Mohammadi, S. E., \& Mohammadi, E. (2018). Robust portfolio optimization based on minimax regret approach in Tehran stock exchange market. Journal of Industrial and Systems Engineering, 11(Special issue: 14th International Industrial Engineering Conference), 51-62.

Ni, G., Feng, X., \& Chen, L. (2019). Hotel room allocation with multiple sales channels: A perspective of minimax regret. Journal of Control and Decision, 7, 1-20.

Perakis, G., \& Roels, G. (2008). Regret in the newsvendor model with partial information. Operations Research, 56(1), 188-203.

Perakis, G., \& Roels, G. (2010). Robust controls for network revenue management. Manufacturing \& Service Operations Management, 12(1), 56-76.

Perakis, G., \& Sood, A. (2006). Competitive multi-period pricing for perishable products: A robust optimization approach. Mathematical Programming, 107(1-2), 295-335.

Popescu, A., Keskinocak, P., Johnson, E., LaDue, M., \& Kasilingam, R. (2006). Estimating air-cargo overbooking based on a discrete show-up-rate distribution. Interfaces, 36(3), 248-258.

Qin, C. R., Luo, L., You, Y., \& Xiao, Y. X. (2012). An optimization model of the single-leg air cargo space control based on Markov decision process. Journal of Applied Mathematics, 2012, 1.

Qu, H., Ryzhov, I. O., Fu, M. C., Bergerson, E., Kurka, M., \& Kopacek, L. (2020). Learning demand curves in B2B pricing: A new framework and case study. Production and Operations Management, 29(5), 1287-1306.

Razin, A. (1976). Rational insurance purchasing. The Journal of Finance, 31(1), 133-137.

Savage, L. J. (1972). The foundations of statistics. North Chelmsford: Courier Corporation.

Stoye, J. (2005). Statistical decisions under ambiguity: An axiomatic analysis. Technical report Northwestern University.

Wald, A. (1950). Statistical decision functions. New York: Wiley. 
Wang, J., \& Xiao, B. (2017). A minmax regret price control model for managing perishable products with uncertain parameters. European Journal of Operational Research, 258(2), 652-663.

Wang, Y. J., \& Kao, C. S. (2008). An application of a fuzzy knowledge system for air cargo overbooking under uncertain capacity. Computers \& Mathematics with Applications, 56(10), 2666-2675.

Wang, Z., Deng, S., \& Ye, Y. (2014). Close the gaps: A learning-while-doing algorithm for single-product revenue management problems. Operations Research, 62(2), 318-331.

Xidonas, P., Mavrotas, G., Hassapis, C., \& Zopounidis, C. (2017). Robust multiobjective portfolio optimization: A minimax regret approach. European Journal of Operational Research, 262(1), 299-305.

Yang, C. H., Ma, X., \& Talluri, S. (2019). Optimal acquisition decision in a remanufacturing system with partial random yield information. International Journal of Production Research, 57(6), 1624-1644.

Yue, J., Chen, B., \& Wang, M. C. (2006). Expected value of distribution information for the newsvendor problem. Operations research, 54(6), 1128-1136.

Publisher's Note Springer Nature remains neutral with regard to jurisdictional claims in published maps and institutional affiliations. 